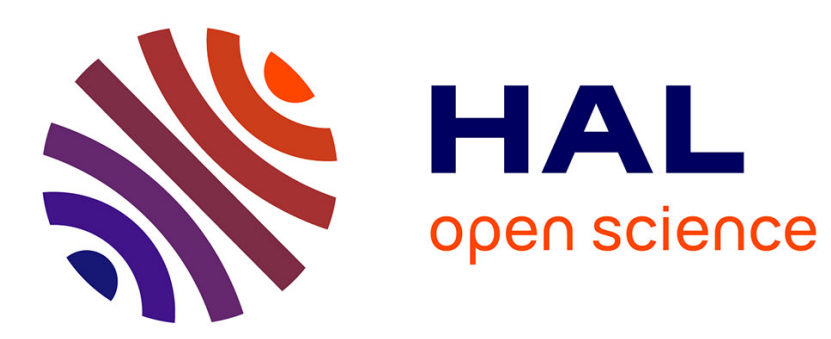

\title{
Robust a posteriori error control and adaptivity for multiscale, multinumerics, and mortar coupling
}

Gergina Pencheva, Martin Vohralík, Mary Wheeler, Tim Wildey

\section{To cite this version:}

Gergina Pencheva, Martin Vohralík, Mary Wheeler, Tim Wildey. Robust a posteriori error control and adaptivity for multiscale, multinumerics, and mortar coupling. SIAM Journal on Numerical Analysis, 2013, 51 (1), pp.526-554. 10.1137/110839047 . hal-00467738v2

\section{HAL Id: hal-00467738 \\ https://hal.science/hal-00467738v2}

Submitted on 7 Jul 2011

HAL is a multi-disciplinary open access archive for the deposit and dissemination of scientific research documents, whether they are published or not. The documents may come from teaching and research institutions in France or abroad, or from public or private research centers.
L'archive ouverte pluridisciplinaire HAL, est destinée au dépôt et à la diffusion de documents scientifiques de niveau recherche, publiés ou non, émanant des établissements d'enseignement et de recherche français ou étrangers, des laboratoires publics ou privés. 


\title{
ROBUST A POSTERIORI ERROR CONTROL AND ADAPTIVITY FOR MULTISCALE, MULTINUMERICS, AND MORTAR COUPLING*
}

\author{
GERGINA V. PENCHEVA ${ }^{\dagger}$, MARTIN VOHRALÍK ${ }^{\ddagger}$, MARY F. WHEELER ${ }^{\dagger}$, AND TIM \\ WILDEY§
}

\begin{abstract}
We consider discretizations of a model elliptic problem by means of different numerical methods applied separately in different subdomains, termed multinumerics, coupled using the mortar technique. The grids need not match along the interfaces. We are also interested in the multiscale setting, where the subdomains are partitioned by a mesh of size $h$, whereas the interfaces are partitioned by a mesh of much coarser size $H$, and where lower-order polynomials are used in the subdomains and higher-order polynomials are used on the mortar interface mesh. We derive several fully computable a posteriori error estimates which deliver a guaranteed upper bound on the error measured in the energy norm. Our estimates are also locally efficient and one of them is robust with respect to the ratio $H / h$ under an assumption of sufficient regularity of the weak solution. The present approach allows bounding separately and comparing mutually the subdomain and interface errors. A subdomain/interface adaptive refinement strategy is proposed and numerically tested.
\end{abstract}

Key words. multiscale, multinumerics, mortar coupling, nonmatching grids, a posteriori error estimate, guaranteed upper bound, robustness, balancing error components

AMS subject classifications. 65N15, 65N30, 76S05

1. Introduction. We consider in this paper the model problem

$$
-\nabla \cdot(\mathbf{K} \nabla p)=f \text { in } \Omega, \quad p=0 \text { on } \partial \Omega,
$$

where $\Omega \subset \mathbb{R}^{d}, d=2,3$, is a polygonal (polyhedral) domain (open, bounded, and connected set), $\mathbf{K}$ is a symmetric, bounded, and uniformly positive definite tensor, and $f \in L^{2}(\Omega)$. The problem (1.1) can be equivalently written as the first order system

$$
\mathbf{u}=-\mathbf{K} \nabla p \text { in } \Omega, \quad \nabla \cdot \mathbf{u}=f \text { in } \Omega, \quad p=0 \text { on } \partial \Omega .
$$

We note that $\mathbf{u}$ is the weak flux satisfying $\mathbf{u} \in \mathbf{H}(\operatorname{div}, \Omega)$, where $\mathbf{H}(\operatorname{div}, \Omega):=\{\mathbf{v} \in$ $\left.\mathbf{L}^{2}(\Omega): \nabla \cdot \mathbf{v} \in L^{2}(\Omega)\right\}$. We are interested in discretizations of (1.1) (or (1.2)) by different numerical methods in the different subdomains $\Omega_{i}$ of $\Omega$. The coupling of these different methods is achieved by the mortar technique. We allow for the cases where the grids of the individual subdomains do not match along the interfaces and

${ }^{*}$ A portion of this research was supported by the U.S. Department of Energy, Office of Science, Office of Basic Energy Sciences. The Center for Frontiers of Subsurface Energy Security (CFSES) is a DOE Energy Frontier Research Center, under Contract No. DE-SC0001114. The authors gratefully acknowledge the financial support provided by the NSF-CDI under contract number DMS 0835745 and King Abdullah University of Science and Technology (KAUST)-AEA-UTA08-687 and DOE grant DE-FGO2-04ER25617. The second author was supported by the GNR MoMaS project "Numerical Simulations and Mathematical Modeling of Underground Nuclear Waste Disposal", PACEN/CNRS, ANDRA, BRGM, CEA, EdF, IRSN, France.

${ }^{\dagger}$ Institute for Computational Engineering and Sciences, University of Texas at Austin, USA (gergina@ices.utexas.edu, mfw@ices.utexas.edu).

$¥$ UPMC Univ. Paris 06, UMR 7598, Laboratoire Jacques-Louis Lions, 75005, Paris, France \& CNRS, UMR 7598, Laboratoire Jacques-Louis Lions, 75005, Paris, France (vohralik@ann.jussieu.fr).

$\S$ Sandia National Labs, Albuquerque, NM 87185. Sandia National Laboratories is a multiprogram laboratory managed and operated by Sandia Corporation, a wholly owned subsidiary of Lockheed Martin Corporation, for the U.S. Department of Energy's National Nuclear Security Administration under contract DE-AC04-94AL85000 (tmwilde@sandia.gov). 
where the subdomain grid elements are a collection of simplices and rectangular parallelepipeds for $d=2$. We will call such grids hybrid. We also investigate the case where the size of the subdomain grids, say $h$, is much smaller than the size of the interface grid, say $H$. More precisely, we allow that $H=\mathcal{O}\left(h^{\beta}\right)$ with $\beta<1$; then lower-order polynomials are used in the subdomain grids and higher-order polynomials are used on the mortar interface mesh. Particular examples of such discretizations are the multiscale mortar mixed finite element method proposed in [8] or the multiscale mortar coupled mixed finite element-discontinuous Galerkin method of [19]. Note that multiscale mortar techniques are especially appealing as the discretization can be reduced to a global problem only involving higher-order polynomials on the interface mortar mesh (see [8]). This leads to a parallel domain decomposition implementation [20] that can be enhanced by constructing a multiscale mortar basis as in [18] and applying multiscale preconditioners [34]. This approach leads to high computational efficiency.

For previous work on a posteriori error estimates for multiscale discretizations, we refer to $[25,1,23]$ and to the references therein. A posteriori estimates for discretizations with mortar coupling have been analyzed in $[36,10,13,11]$ for conforming and nonconforming Galerkin methods and in $[35,8]$ for mixed finite element methods; and a posteriori estimates for multinumerics in $[11,15]$. Our approach is based on potential and flux reconstruction and develops ideas going back to at least the PragerSynge equality [27]. These ideas have been recently used in $[22,31,4,16,14,17,5]$, see also the references therein, but have not been applied to the case of multiscale mortar discretizations.

The purpose of this paper is to derive a general and unified framework for optimal a posteriori error estimation in the multiscale, multinumerics, and mortar coupling setting. We derive several fully and locally computable estimates providing a guaranteed upper bound on the energy error. Our estimators are also locally efficient, giving local lower bounds on the energy error. Importantly, this property holds independently of the use of different discretization schemes in different parts of the domain, of the use of the mortar coupling, and, to a reasonable degree, of the non-alignment of the subdomain meshes at the interfaces. Our estimates are thus robust with respect to the multinumerics and mortar coupling. Moreover, one of them gives estimates robust with respect to the ratio $H / h$ and is thus robust with respect to the multiscale.

The paper is organized as follows. In Section 2, we set up the notation, define the admissible grids and finite-dimensional spaces, and describe the continuous setting. Our a posteriori error estimates are stated and proved in Section 3. We do so in a general formulation, not mentioning any particular (combination of) approaches employed. We only suppose that we are given an approximate flux $\mathbf{u}_{h}$ which is $\mathbf{H}$ (div)conforming inside each subdomain $\Omega_{i}$, locally conservative inside each subdomain $\Omega_{i}$, and whose normal trace is weakly continuous across the interface; see Assumption 3.7. As mentioned earlier, we derive several estimators. The first two, detailed in Section 3, rely on a construction of a globally $\mathbf{H}(\operatorname{div}, \Omega)$-conforming flux $\mathbf{t}_{h}$ by solution of local Neumann problems using mixed finite element methods. The first one requires a construction of a matching $h$-sized grid $\widehat{\mathcal{T}}_{h}$ of $\Omega$ and the local problems are $H$-sized with lower-order polynomial degrees. The second one requires a construction of a matching $H$-sized grid $\mathcal{T}_{H}$ of $\Omega$ and the local problems are $\Omega_{i}$-sized with higher-order polynomial degrees.

Section 4 investigates the local efficiency of the derived estimates. Once again, this is done generally, without a specification of the underlying numerical schemes; we only need to assume the weak continuity of the approximate potential $\tilde{p}_{h}$ (Assumption 3.8). Section 5 then gives examples of multiscale, multinumerics, and mortar 

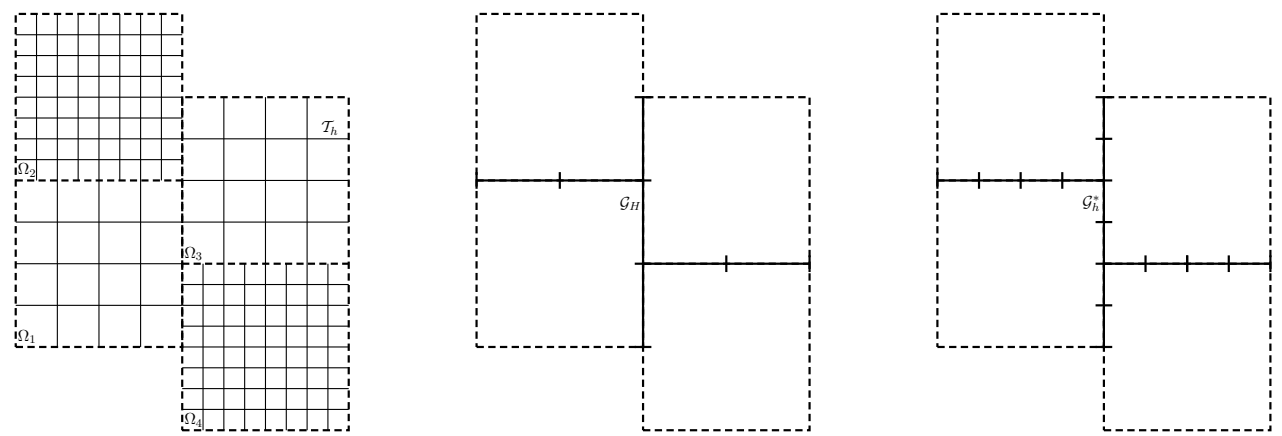

FIG. 2.1. Example of a domain $\Omega$ with subdomains $\Omega_{i}$ and nonmatching mesh $\mathcal{T}_{h}$ (left), interface mesh $\mathcal{G}_{H}$ (middle), and the corresponding interface mesh $\mathcal{G}_{h}^{*}$ (right)

discretizations. We therein also verify Assumptions 3.7 and 3.8 for each example in question. We sketch in Section 6 our last, alternative estimator, only relying on the given (nonmatching) grid $\mathcal{T}_{h}$ and not requiring any construction of a globally $\mathbf{H}(\operatorname{div}, \Omega)$-conforming flux $\mathbf{t}_{h}$. In all of our estimates we distinguish and estimate separately the errors arising inside of the subdomains and those emanating from the mortar coupling. In Section 7 we present an adaptive algorithm that balances the two error contributions and provide numerical experiments illustrating the theoretical developments. Finally, Appendix A gives a technical result necessary for the analysis on nonmatching grids.

2. Preliminaries. We introduce in this section the partitions of $\Omega$, notation, weak solutions, and energy norms.

Let $D \subset \Omega$. Then $\|\cdot\|_{D}$ stands for the $L^{2}(D)$ norm and $(\cdot, \cdot)_{D}$ for the $L^{2}(D)$ scalar product. When $D$ coincides with $\Omega$, the subscript $D$ is dropped. The $L^{2}(D)$ scalar product for $D \subset \mathbb{R}^{d-1}$ will be denoted by $\langle\cdot, \cdot\rangle_{D}$. We also use the notation $|D|$ for the $d^{\prime}$-dimensional Lebesgue measure of $D \subset \mathbb{R}^{d^{\prime}}, 1 \leq d^{\prime} \leq d$. For $v \in L^{1}(D)$, we denote by $v_{D}$ the mean value of $v$ on $D$. For $D \subset \Omega, c_{\mathbf{K}, D}, C_{\mathbf{K}, D}$ stand for the smallest and largest eigenvalue of $\mathbf{K}$ on $D$, respectively.

2.1. Partitions of $\Omega$ and of $\Gamma$. We suppose that $\Omega$ is decomposed into nonoverlapping polygonal (polyhedral) subdomains $\Omega_{i}, i \in\{1, \ldots, n\}$. This partition can be nonmatching in the sense that neighboring subdomains need not share complete sides (edges if $d=2$, faces if $d=3$ ). We denote $\Gamma_{i, j}:=\partial \Omega_{i} \cap \partial \Omega_{j}, \Gamma:=\cup_{1 \leq i<j \leq n} \Gamma_{i, j}$, and $\Gamma_{i}:=\partial \Omega_{i} \cap \Gamma$. Let $\mathcal{T}_{h, i}$ be a matching finite element mesh of $\Omega_{i}, i \in\{1, \ldots, n\}$, composed of (closed) simplices and/or rectangular parallelepipeds. A collection of triangles and rectangles is allowed for $d=2$ but we only allow for either tetrahedra or rectangular parallelepipeds for $d=3$. We would need to introduce other elements like prisms for $d=3$ in order to allow for general hybrid grids, which we prefer to avoid for the sake of simplicity. We then set $\mathcal{T}_{h}:=\cup_{i=1}^{n} \mathcal{T}_{h, i}$ and denote by $h$ the maximal element diameter in $\mathcal{T}_{h}$; note that $\mathcal{T}_{h}$ can be nonmatching as neighboring meshes $\mathcal{T}_{h, i}$ and $\mathcal{T}_{h, j}$ need not align on $\Gamma_{i, j}$. A generic element of the partition $\mathcal{T}_{h}$ will be denoted by $T ; h_{T}$ stands for the diameter of $T$. This setting is illustrated in Figure 2.1 (left).

We use $\mathcal{E}_{h, i}^{\text {int }}$ to denote the interior sides of $\mathcal{T}_{h, i}, i \in\{1, \ldots, n\}$, and set $\mathcal{E}_{h}^{\text {int }}:=$ $\cup_{i=1}^{n} \mathcal{E}_{h, i}^{\text {int }} ; \mathcal{E}_{h}^{\text {int }}$ thus contains neither the subdomain interfaces nor the outer boundary of $\Omega$. We denote by $\mathcal{E}_{h, i}$ all the sides of $\mathcal{T}_{h, i}$ and set $\mathcal{E}_{h}:=\cup_{i=1}^{n} \mathcal{E}_{h, i}$. We let $\mathcal{E}_{h, i, j}^{\Gamma}$ be the partition of $\Gamma_{i, j}$ by the sides of $\mathcal{T}_{h, i}$ and $\mathcal{E}_{h, i}^{\Gamma}$ the partition of $\Gamma_{i}$ by the sides of $\mathcal{T}_{h, i}$. We denote by $\mathcal{E}_{h}^{\Gamma}:=\cup_{1 \leq i \leq n} \mathcal{E}_{h, i}^{\Gamma}$ all the sides of $\mathcal{T}_{h}$ located at the interface $\Gamma$ and 

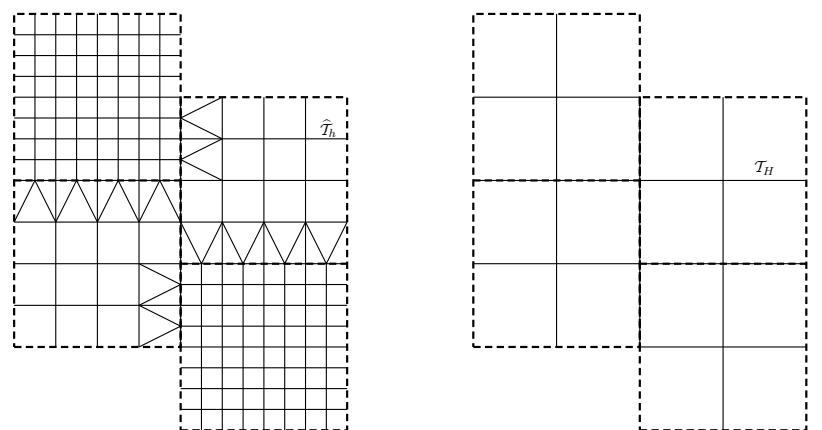

FIG. 2.2. Example of a matching refinement $\widehat{\mathcal{T}}_{h}$ of $\mathcal{T}_{h}$ (left) and of a mesh $\mathcal{T}_{H}$ (right)

by $\mathcal{E}_{h}^{\text {ext }}:=\mathcal{E}_{h} \backslash \mathcal{E}_{h}^{\text {int }} \backslash \mathcal{E}_{h}^{\Gamma}$ the faces of $\mathcal{T}_{h}$ located at the boundary of $\Omega$. We also set $\mathcal{E}_{h}^{\mathrm{int}, \Gamma}:=\mathcal{E}_{h}^{\mathrm{int}} \cup \mathcal{E}_{h}^{\Gamma}$. The notation $\mathcal{E}_{T}$ stands for all the sides of an element $T \in \mathcal{T}_{h}$. A generic side of $\mathcal{E}_{h}$ will be denoted by $e$ and its diameter by $h_{e}$.

Next, we let $\mathcal{G}_{H, i, j}$ be the mortar interface finite element mesh of $\Gamma_{i, j}$. The elements $g \in \mathcal{G}_{H, i, j}$ are either line segments (if $d=2$ ) or triangles or rectangles (if $d=3)$. We do not require $\mathcal{G}_{H, i, j}$ to be matching in the sense of a $(d-1)$-dimensional mesh of $\Gamma_{i, j}$. We set $\mathcal{G}_{H, i}:=\cup_{1 \leq j \leq n} \mathcal{G}_{H, i, j}$ and $\mathcal{G}_{H}:=\cup_{1 \leq i<j \leq n} \mathcal{G}_{H, i, j}$, cf. Figure 2.1 (middle). Maximal element diameter in $\mathcal{G}_{H}$ is denoted by $H$. In the multiscale setting, $h<H \leq 1$ and the ratio $H / h$ can be unbounded, $H=\mathcal{O}\left(h^{\beta}\right)$ with $\beta<1$. Note that on an interface $\Gamma_{i, j}, \mathcal{G}_{H, i, j}$ is a unique $(d-1)$-dimensional surface mesh, whereas there are two (different) meshes $\mathcal{E}_{h, i, j}^{\Gamma}$ and $\mathcal{E}_{h, j, i}^{\Gamma}$ from the two sides of the interface. Also, the meshes $\mathcal{E}_{h, i, j}^{\Gamma}$ and $\mathcal{E}_{h, j, i}^{\Gamma}$ need in general not be refinements of $\mathcal{G}_{H, i, j}$; we will, however, need such a requirement for specific cases discussed later. We also assume that the intersection of the meshes $\mathcal{E}_{h, i, j}^{\Gamma}$ and $\mathcal{E}_{h, j, i}^{\Gamma}$ is a matching mesh of $\Gamma_{i, j}$ consisting of line segments (if $d=2$ ) or triangles or rectangles (if $d=3$ ).

We will in the sequel also use the following partition of $\Gamma$. Let an interface $\Gamma_{i, j}$ be given. We define the mesh $\mathcal{G}_{h, i, j}^{*}$ as a set of $(d-1)$-dimensional sides $g$, where each $g \in \mathcal{G}_{h, i, j}^{*}$ is simultaneously a union of sides from $\mathcal{E}_{h, i, j}^{\Gamma}$ and a union of sides from $\mathcal{E}_{h, j, i}^{\Gamma}$. We choose the sides $g$ so as to be composed of the smallest possible number of sides from $\mathcal{E}_{h, i, j}^{\Gamma}$ and $\mathcal{E}_{h, j, i}^{\Gamma}$. There is one mesh $\mathcal{G}_{h, i, j}^{*}$ for each interface $\Gamma_{i, j}$. We set $\mathcal{G}_{h, i}^{*}:=\cup_{1 \leq j \leq n} \mathcal{G}_{h, i, j}^{*}$ and $\mathcal{G}_{h}^{*}:=\cup_{1 \leq i<j \leq n} \mathcal{G}_{h, i, j}^{*}$, cf. Figure 2.1 (right). We denote by $H_{g}$ the diameter of a side $g \in \mathcal{G}_{H}$ and by $h_{g}$ the diameter of a side $g \in \mathcal{G}_{h}^{*}$.

Finally, two other types of partitions of $\Omega$ will be used in the paper, $\widehat{\mathcal{T}}_{h}$ and $\mathcal{T}_{H}$. Let $\widehat{\mathcal{T}}_{h}$ be a matching refinement of $\mathcal{T}_{h}$, consisting of simplices and/or rectangular parallelepipeds; hybrid grids are allowed for $d=2$. We refer to Figure 2.2 (left) for an example of $\widehat{\mathcal{T}}_{h}$. We denote by $\widehat{\mathcal{E}}_{h}$ the sides of $\widehat{\mathcal{T}}_{h}$ and use the notation $\widehat{\mathcal{T}}_{h, i}$ for the restriction of $\widehat{\mathcal{T}}_{h}$ on the subdomain $\Omega_{i}$. We suppose that $\widehat{\mathcal{T}}_{h, i}$ coincides with $\mathcal{T}_{h, i}$ in the interior of each subdomain $\Omega_{i}$ and only differs from $\mathcal{T}_{h}$ near the interfaces. More precisely, we assume that for each $T \in \mathcal{T}_{h}$ such that $T \cap \Gamma=\emptyset$, there exists an element $T^{\prime} \in \widehat{\mathcal{T}}_{h}$ such that $T=T^{\prime}$ and that every side $e^{\prime} \in \widehat{\mathcal{E}}_{h}$ which shares a node with this $T^{\prime}$ either coincides with some $e \in \mathcal{E}_{h}^{\text {int }}$ or belongs to the interior of some $T^{\prime \prime} \in \mathcal{T}_{h}$. We assume that $\widehat{\mathcal{T}}_{h}$ adds no new nodes and sides at the interface $\Gamma$ in comparison with $\mathcal{T}_{h}$. The mesh $\mathcal{T}_{H}$ is in general formed by groups of elements from $\mathcal{T}_{h}$ and is matching in the sense that the restriction of $\mathcal{T}_{H}$ on $\Gamma$ is the interface mesh $\mathcal{G}_{H}$. As before, we use the notation $\mathcal{T}_{H, i}$ for the restriction of $\mathcal{T}_{H}$ on $\Omega_{i}$. We refer to Figure 2.2 (right) for an example of $\mathcal{T}_{H}$. For an element $T \in \mathcal{T}_{H}$, we denote by $\mathcal{G}_{T}$ the set of its sides. 
2.2. Finite-dimensional spaces and projection operators. We begin with the mortar space $M_{H}$. It is the space of discontinuous piecewise polynomials of order $m$ on the interface mesh $\mathcal{G}_{H} ; M_{H}$ thus in particular contains piecewise constant functions on $\mathcal{G}_{H}$. We next define the spaces on $\mathcal{T}_{h}$. If $T \in \mathcal{T}_{h}$ is a simplex, we let $\mathbb{R}_{r}(T):=\mathbb{P}_{r}(T)$ be the space of polynomials of total degree at most $r$. If $T \in \mathcal{T}_{h}$ is a rectangular parallelepiped, we let $\mathbb{R}_{r}(T):=\mathbb{Q}_{r}(T)$ be the space of polynomials of degree at most $r$ in each variable. We then define $\mathbb{R}_{r}\left(\mathcal{T}_{h}\right)$ as the space such that for each $w \in \mathbb{R}_{r}\left(\mathcal{T}_{h}\right),\left.w\right|_{T} \in \mathbb{R}_{r}(T)$; we require no continuity at the sides. We also define $\mathbb{R}_{k-1, *, d}(T)$ by $\left[\mathbb{P}_{k-1}(T)\right]^{d}$ for a simplex and $\mathbb{Q}_{k-1, k}(T) \times \mathbb{Q}_{k, k-1}(T)$ if $d=2$ and $\mathbb{Q}_{k-1, k, k}(T) \times \mathbb{Q}_{k, k-1, k}(T) \times \mathbb{Q}_{k, k, k-1}(T)$ if $d=3$ for a rectangular parallelepiped. We set $\mathbf{R T N}^{k}\left(\mathcal{T}_{h, i}\right):=\left\{\mathbf{v} \in \mathbf{H}\left(\operatorname{div}, \Omega_{i}\right):\left.\mathbf{v}\right|_{T} \in\left[\mathbb{R}_{k}(T)\right]^{d}+\mathbb{R}_{k}(T) \mathbf{x} \quad \forall T \in \mathcal{T}_{h, i}\right\}$.

Let $\mathbf{V}_{h, i} \times W_{h, i} \subset \mathbf{H}\left(\operatorname{div}, \Omega_{i}\right) \times L^{2}\left(\Omega_{i}\right)$ be the Raviart-Thomas-Nédélec (RTN) mixed finite element spaces of order $k, \mathbf{V}_{h, i}:=\mathbf{R T N}^{k}\left(\mathcal{T}_{h, i}\right), W_{h, i}:=\mathbb{R}_{k}\left(\mathcal{T}_{h, i}\right)$, cf. [12, 29]. Our results can be extended to other mixed finite element spaces. We can also take into account spaces with different polynomial degrees in different subdomains and also with different polynomial degrees in different elements; we restrict ourselves to the given setting for the sake of brevity and clarity. We set $\mathbf{V}_{h}:=\bigoplus_{i=1}^{n} \mathbf{V}_{h, i}$, $W_{h}:=\bigoplus_{i=1}^{n} W_{h, i}$. Note that the normal components of vectors in $\mathbf{V}_{h}$ are continuous across the sides between elements in each subdomain $\Omega_{i}$ but not across $\Gamma$. We also define the pairs $\left(\mathbf{V}_{\widehat{h}}, W_{\widehat{h}}\right)$ and $\left(\mathbf{V}_{H}, W_{H}\right)$ as the RTN spaces on the matching submesh $\widehat{\mathcal{T}}_{h}$ of $\mathcal{T}_{h}$ of order $k$ and on the coarse mesh $\mathcal{T}_{H}$ of order $m$, respectively. $V(S)$ stands for the restriction of the space $V$ defined on $\Omega$ (mesh $\mathcal{T}_{h}$ ), to the subdomain (submesh) $S$.

We will also need some orthogonal projections: let $P_{W_{h}}$ be the $L^{2}(\Omega)$-orthogonal projection onto $W_{h}, P_{W_{\widehat{h}}}$ the $L^{2}(\Omega)$-orthogonal projection onto $W_{\widehat{h}}, P_{W_{H}}$ the $L^{2}(\Omega)$ orthogonal projection onto $W_{H}$, and $P_{M_{H}}$ the $L^{2}(\Gamma)$-orthogonal projection onto $M_{H}$,

$$
\begin{array}{llll}
P_{W_{h}}: L^{2}(\Omega) \rightarrow W_{h} & \text { for } w \in L^{2}(\Omega), & \left(w-P_{W_{h}}(w), w_{h}\right)=0 & \forall w_{h} \in W_{h}, \\
P_{W_{\widehat{h}}}: L^{2}(\Omega) \rightarrow W_{\widehat{h}} & \text { for } w \in L^{2}(\Omega), & \left(w-P_{W_{\widehat{h}}}(w), w_{h}\right)=0 & \forall w_{h} \in W_{\widehat{h}}, \\
P_{W_{H}}: L^{2}(\Omega) \rightarrow W_{H} & \text { for } w \in L^{2}(\Omega), & \left(w-P_{W_{H}}(w), w_{H}\right)=0 & \forall w_{H} \in W_{H}, \\
P_{M_{H}}: L^{2}(\Gamma) \rightarrow M_{H} & \text { for } \mu \in L^{2}(\Gamma), & \left(\mu-P_{M_{H}}(\mu), \mu_{H}\right)_{\Gamma}=0 & \forall \mu_{H} \in M_{H} .
\end{array}
$$

We also denote by $\pi_{l}$ the orthogonal projection onto $\mathbb{R}_{l}(\mathcal{T})$ where $\mathcal{T}$ is the given mesh.

2.3. Other notation. Let $H^{1}\left(\mathcal{T}_{h}\right):=\left\{\varphi \in L^{2}(\Omega):\left.\varphi\right|_{T} \in H^{1}(T) \forall T \in \mathcal{T}_{h}\right\}$ be the broken Sobolev space. We will use the $\operatorname{sign} \nabla$ to denote the elementwise gradient.

For a sufficiently smooth function $v$ that is double-valued on an interior side $e \in \mathcal{E}_{h}^{\text {int }}, e=T^{-} \cap T^{+}$, its jump and average on $e$ are defined as

$$
\llbracket v \rrbracket:=\left.v\right|_{T^{-}}-\left.v\right|_{T^{+}}, \quad\{v v\}:=\frac{1}{2}\left(\left.v\right|_{T^{-}}+\left.v\right|_{T^{+}}\right) .
$$

We denote $\mathbf{n}_{e}$ the unit normal vector, pointing from $T^{-}$towards $T^{+}$. For $e \in \mathcal{E}_{h}^{\text {ext }}$, we set $\llbracket v \rrbracket:=\left.v\right|_{e}$ and $\{\{v\}\}:=\left.v\right|_{e}$. We use similar notation for the sides $g$ from $\mathcal{G}_{H}$ and $\mathcal{G}_{h}^{*}$ and also for the sides $e$ from $\widehat{\mathcal{E}}_{h}$. For boundary sides $e, \mathbf{n}_{e}$ coincides with the unit normal vector, outward to $\partial \Omega$. Similarly, $\mathbf{n}_{\Gamma}$ stands for the unit normal vector to $\Gamma$, with arbitrary but fixed orientation, and for a subset $D$ of $\Omega, \mathbf{n}_{D}$ is used to denote the unit normal vector, outward to $\partial D$.

We will utilize below Poincaré's inequality. For an element $T$, there exist a constant $C_{\mathrm{P}, T}$ such that

$$
\left\|\varphi-\varphi_{T}\right\|_{T} \leq C_{\mathrm{P}, T} h_{T}\|\nabla \varphi\|_{T} \quad \forall \varphi \in H^{1}(T) .
$$


The constant $C_{\mathrm{P}, T}$ is equal to $1 / \pi$ when $T$ is convex.

2.4. Weak solution and energy norm. Let the symmetric bilinear form $\mathcal{A}$ be given by

$$
\mathcal{A}(\mathbf{z}, \mathbf{v}):=\left(\mathbf{z}, \mathbf{K}^{-1} \mathbf{v}\right), \quad \mathbf{z}, \mathbf{v} \in \mathbf{L}^{2}(\Omega) .
$$

The weak solution of (1.1) is a function $p \in H_{0}^{1}(\Omega)$ such that

$$
\mathcal{A}(\mathbf{K} \nabla p, \mathbf{K} \nabla \varphi)=(f, \varphi) \quad \forall \varphi \in H_{0}^{1}(\Omega) .
$$

We define the energy seminorm on $H^{1}\left(\mathcal{T}_{h}\right)$

$$
\|\varphi\|^{2}:=\mathcal{A}(\mathbf{K} \nabla \varphi, \mathbf{K} \nabla \varphi)=\left\|\mathbf{K}^{\frac{1}{2}} \nabla \varphi\right\|^{2}, \quad \varphi \in H^{1}\left(\mathcal{T}_{h}\right),
$$

and the energy norm on $\mathbf{L}^{2}(\Omega)$ by

$$
\|\mathbf{v}\|_{*}^{2}:=\mathcal{A}(\mathbf{v}, \mathbf{v})=\left\|\mathbf{K}^{-\frac{1}{2}} \mathbf{v}\right\|^{2}, \quad \mathbf{v} \in \mathbf{L}^{2}(\Omega) .
$$

3. A posteriori error estimates. We present in this section a framework for a posteriori error estimates in the multiscale, multinumerics, and mortar coupling setting. Our framework does not rely on any particular discretization; applications to different methods are presented in Section 5 below.

3.1. Estimates for the flux. We first study the error in the approximate flux $\mathbf{u}_{h}$. Let $\mathcal{T}_{\hbar}$ be either $\widehat{\mathcal{T}}_{h}$ or $\mathcal{T}_{H}$, to be determined later. Our upper bound result is:

TheOrEm 3.1 (Estimate for the flux). Let $\mathbf{u}$ be the exact flux defined by (1.2) and let $\mathbf{u}_{h} \in \mathbf{L}^{2}(\Omega)$ be arbitrary. For $s_{h} \in H_{0}^{1}(\Omega)$ and $\mathbf{t}_{h} \in \mathbf{H}(\operatorname{div}, \Omega)$ satisfying

$$
\left(\nabla \cdot \mathbf{t}_{h}, 1\right)_{T}=(f, 1)_{T} \quad \forall T \in \mathcal{T}_{\hbar},
$$

we have

$$
\left\|\mid \mathbf{u}-\mathbf{u}_{h}\right\|_{*} \leq \eta_{\mathrm{P}}+\eta_{\mathrm{M}}+\eta_{\mathrm{R}, \hbar}
$$

where the potential, mortar, and residual estimators are given respectively by

$$
\begin{aligned}
\eta_{\mathrm{P}} & :=\left\|\mathbf{u}_{h}+\mathbf{K} \nabla s_{h}\right\|_{*}, \\
\eta_{\mathrm{M}} & :=\left\|\mid \mathbf{u}_{h}-\mathbf{t}_{h}\right\|_{*}, \\
\eta_{\mathrm{R}, \hbar} & :=\left\{\sum_{T \in \mathcal{T}_{\hbar}} C_{\mathrm{P}, T}^{2} \hbar_{T}^{2} c_{\mathbf{K}, T}^{-1}\left\|f-\nabla \cdot \mathbf{t}_{h}\right\|_{T}^{2}\right\}^{\frac{1}{2}} .
\end{aligned}
$$

Proof. It follows readily from Green's theorem and [33, Theorems 3.1 and 6.1] that

$$
\begin{aligned}
\left\|\mathbf{u}-\mathbf{u}_{h} \mid\right\|_{*} \leq & \inf _{s \in H_{0}^{1}(\Omega)}\left\|\mathbf{u}_{h}+\mathbf{K} \nabla s\right\|_{*} \\
& +\inf _{\mathbf{t} \in \mathbf{H}(\operatorname{div}, \Omega)}\left\{\left\|\mathbf{u}_{h}-\mathbf{t}\right\|_{*}+\sup _{\varphi \in H_{0}^{1}(\Omega),\|\mid \varphi\| \|=1}(f-\nabla \cdot \mathbf{t}, \varphi)\right\} .
\end{aligned}
$$

The first two terms in (3.6) yield the $\eta_{\mathrm{P}}$ and the $\eta_{\mathrm{M}}$ estimators (3.3) and (3.4). Let $\varphi \in H_{0}^{1}(\Omega)$ with $\|\varphi\|=1$. By (3.1) and using the Cauchy-Schwarz's inequality, Poincaré's inequality (2.3), and the definition of the ||$|\cdot|||$ norm (2.6), we have

$$
\left(f-\nabla \cdot \mathbf{t}_{h}, \varphi\right)=\sum_{T \in \mathcal{T}_{\hbar}}\left(f-\nabla \cdot \mathbf{t}_{h}, \varphi-\varphi_{T}\right)_{T} \leq \eta_{\mathrm{R}, \hbar}\left|\|\varphi \mid\|=\eta_{\mathrm{R}, \hbar},\right.
$$


which finishes the proof.

We will call the function $s_{h}$ the potential reconstruction and the function $\mathbf{t}_{h}$ the flux reconstruction. Their practical construction is given in Section 3.3 below.

REMARK 3.2 (Estimating the mortar error). The mortar estimator $\eta_{\mathrm{M}}$ differs significantly from previous work, cf. [35, 8], where it takes the form (6.1). The present $\eta_{\mathrm{M}}$ allows for an optimal upper bound and robustness in a multiscale setting.

3.2. Estimates for the potential. We state here our estimates for the error in the approximate potential $\tilde{p}_{h}$. Let $\mathcal{T}_{\hbar}$ be as above.

TheOREM 3.3 (Estimate for the potential). Let $p$ be the exact potential defined by (1.1) and let $\tilde{p}_{h} \in H^{1}\left(\mathcal{T}_{h}\right)$ be arbitrary. For $s_{h} \in H_{0}^{1}(\Omega)$ and $\mathbf{t}_{h} \in \mathbf{H}(\operatorname{div}, \Omega)$ satisfying (3.1), we obtain

$$
\left\|p-\tilde{p}_{h} \mid\right\| \leq \eta_{\mathrm{NC}}+\eta_{\mathrm{DFM}}+\eta_{\mathrm{R}, \hbar},
$$

where $\eta_{\mathrm{R}, \hbar}$ is given by (3.5) and the nonconformity and diffusive flux-mortar estimators are defined as

$$
\begin{array}{r}
\eta_{\mathrm{NC}}:=\mid\left\|\tilde{p}_{h}-s_{h}\right\|, \\
\eta_{\mathrm{DFM}}:=\mid\left\|\mathbf{K} \nabla \tilde{p}_{h}+\mathbf{t}_{h}\right\|_{*} .
\end{array}
$$

Proof. It follows from [31, Lemma 7.1] and Green's theorem that

$$
\begin{aligned}
\left\|p-\tilde{p}_{h}\right\| \mid \leq & \inf _{s \in H_{0}^{1}(\Omega)}\left\|\tilde{p}_{h}-s \mid\right\| \\
& +\inf _{\mathbf{t} \in \mathbf{H}(\operatorname{div}, \Omega)} \sup _{\varphi \in H_{0}^{1}(\Omega),\|\varphi\| \|=1}\left((f-\nabla \cdot \mathbf{t}, \varphi)-\left(\mathbf{K} \nabla \tilde{p}_{h}+\mathbf{t}, \nabla \varphi\right)\right) .
\end{aligned}
$$

Inequality (3.8) is straightforward using (3.7) and Cauchy-Schwarz's inequality.

REMARK 3.4 (Constitutive relation, equilibrium, and constraints). We observe that the estimates of Theorems 3.1 and 3.3 provide estimates of the error in the constitutive relation $\mathbf{u}_{h}=-\mathbf{K} \nabla \tilde{p}_{h}$ with constraints $\mathbf{u}_{h} \in \mathbf{H}(\operatorname{div}, \Omega)$ and $\tilde{p}_{h} \in H_{0}^{1}(\Omega)$ and the equilibrium condition $\nabla \cdot \mathbf{u}_{h}=f$ on the discrete level. In this respect, they are closely related to the Prager-Synge equality [27].

REMARK 3.5 (Subdomain discretization and mortar errors). Let

$$
\eta_{\mathrm{DF}}:=\mid\left\|\mathbf{K} \nabla \tilde{p}_{h}+\mathbf{u}_{h}\right\| \|_{*} .
$$

The triangle inequality yields $\eta_{\mathrm{DFM}} \leq \eta_{\mathrm{DF}}+\eta_{\mathrm{M}}$. It should be noted that $\eta_{\mathrm{P}}, \eta_{\mathrm{R}, \hbar}$, $\eta_{\mathrm{NC}}$, and $\eta_{\mathrm{DF}}$ represent the subdomain error and $\eta_{\mathrm{M}}$ the interface mortar error.

REMARK 3.6 (Residual estimator $\eta_{\mathrm{R}, \hbar}$ ). In Section 3.3.2 we consider $\mathcal{T}_{\hbar}:=\widehat{\mathcal{T}}_{h}$ and $\nabla \cdot \mathbf{t}_{h}=P_{W_{\hat{h}}}(f)$. Here $\eta_{\mathrm{R}, \hbar}$ takes the form of the usual data oscillation estimate on the mesh $\widehat{\mathcal{T}}_{h}$, vanishes whenever $f \in \mathbb{R}_{k}\left(\widehat{\mathcal{T}}_{h}\right)$, and is higher-order convergent whenever $f \in H^{k+1}\left(\widehat{\mathcal{T}}_{h}\right)$. Similar observations can be made for Section 3.3 .3 with $\mathcal{T}_{\hbar}:=\mathcal{T}_{H}$.

3.3. Practical construction of $H_{0}^{1}(\Omega)$ - and $\mathbf{H}(\operatorname{div}, \Omega)$-conforming potential and flux reconstructions. We describe here practical constructions of a $H_{0}^{1}(\Omega)$ conforming potential reconstruction $s_{h}$ and $\mathbf{H}(\operatorname{div}, \Omega)$-conforming flux reconstruction $\mathbf{t}_{h}$ assumed in Theorems 3.1 and 3.3. We propose one method of constructing $s_{h}$ and two methods for constructing $\mathbf{t}_{h}$. In order to proceed generally, we make the following assumptions:

Assumption 3.7 (Properties of $\mathbf{u}_{h}$ ). Let

(1) $\mathbf{u}_{h} \in \mathbf{V}_{h}$; 
(2) $\left(\nabla \cdot \mathbf{u}_{h}, 1\right)_{T}=(f, 1)_{T} \quad \forall T \in \mathcal{T}_{h}$;

(3) $\sum_{i=1}^{n}\left\langle\mathbf{u}_{h} \cdot \mathbf{n}_{\Omega_{i}}, \mu_{H}\right\rangle_{\Gamma_{i}}=0 \quad \forall \mu_{H} \in M_{H}$.

Assumption 3.7 describes general locally conservative multiscale mortar discretizations that also allow for multinumerics [8, 19]. More precisely, we assume: (1) the approximate flux $\mathbf{u}_{h}$ belongs to the RTN space inside each subdomain $\Omega_{i} ;(2) \mathbf{u}_{h}$ is locally conservative inside each subdomain $\Omega_{i}$ on the elements of $\mathcal{T}_{h, i}$; and (3) normal trace of $\mathbf{u}_{h}$ is weakly continuous across the interfaces.

Let $g$ be a mortar element with $g \in \mathcal{G}_{H, i, j}$. Since $M_{H}$ is a space of discontinuous piecewise polynomials, Assumption 3.7 (3) implies that

$$
\left\langle\llbracket \mathbf{u}_{h} \cdot \mathbf{n}_{g} \rrbracket, \mu_{g}\right\rangle_{g}=0 \quad \forall \mu_{g} \in M_{H}(g) .
$$

We define $F \in M_{H}$ such that

$$
\left.F\right|_{\Gamma_{i, j}}:=P_{M_{H}}\left(\left.\left(\left.\mathbf{u}_{h}\right|_{\Omega_{i}} \cdot \mathbf{n}_{\Gamma}\right)\right|_{\Gamma_{i, j}}\right)=P_{M_{H}}\left(\left.\left(\left.\mathbf{u}_{h}\right|_{\Omega_{j}} \cdot \mathbf{n}_{\Gamma}\right)\right|_{\Gamma_{i, j}}\right) \quad i, j \in\{1, \ldots, n\} .
$$

Using weak continuity, we clearly have

$$
\begin{aligned}
\left\langle\left.\mathbf{u}_{h}\right|_{\Omega_{i}} \cdot \mathbf{n}_{g}, 1\right\rangle_{g}= & \left\langle\left.\mathbf{u}_{h}\right|_{\Omega_{j}} \cdot \mathbf{n}_{g}, 1\right\rangle_{g}=\left\langle\left\{\left\{\mathbf{u}_{h} \cdot \mathbf{n}_{g}\right\}, 1\right\rangle_{g}=\langle F, 1\rangle_{g}\right. \\
& \forall g \in \mathcal{G}_{H, i, j}, i, j \in\{1, \ldots, n\} .
\end{aligned}
$$

Assumption 3.8 (Properties of $\tilde{p}_{h}$ ). Let

(1) $\tilde{p}_{h} \in \mathbb{R}_{r}\left(\mathcal{T}_{h}\right)$ for some $r \geq 1$;

(2) $\left\langle\llbracket \tilde{p}_{h} \rrbracket, 1\right\rangle_{e}=0 \quad \forall e \in \mathcal{E}_{h}^{\text {int }} \cup \mathcal{E}_{h}^{\text {ext }}$;

(3) $\left\langle\llbracket \tilde{p}_{h} \rrbracket, 1\right\rangle_{g}=0 \quad \forall g \in \mathcal{G}_{h}^{*}$.

Assumption 3.8 implies: (1) $\tilde{p}_{h}$ is a piecewise polynomial; (2) means of the traces of $\tilde{p}_{h}$ on interior subdomain sides are continuous and means of the traces of $\tilde{p}_{h}$ on boundary sides are zero; (3) means of the traces of $\tilde{p}_{h}$ on collections of sides inside the interface $\Gamma$ are continuous.

3.3.1. Construction of $s_{h}$. We propose here a particular construction of $s_{h}$ relying on the mesh $\widehat{\mathcal{T}}_{h}$; other constructions, using only the mesh $\mathcal{T}_{h}$, are possible.

Recall that $\widehat{\mathcal{T}}_{h}$ is the conforming refinement of $\mathcal{T}_{h}$ introduced in Section 2.1 and note that $\mathbb{R}_{r}\left(\mathcal{T}_{h}\right) \subset \mathbb{R}_{r^{\prime}}\left(\widehat{\mathcal{T}}_{h}\right), r^{\prime} \geq r$, i.e., every piecewise polynomial on $\mathcal{T}_{h}$ is also a piecewise polynomial on $\widehat{\mathcal{T}}_{h}$, where possibly the polynomial degree $r$ is increased to $r^{\prime}$. We can apply the averaging interpolation of $[2,21,32]$. Here $\mathcal{I}_{\text {av }}: \mathbb{R}_{r^{\prime}}\left(\widehat{\mathcal{T}}_{h}\right) \rightarrow$ $\mathbb{R}_{r^{\prime}}\left(\widehat{\mathcal{T}}_{h}\right) \cap H_{0}^{1}(\Omega)$ is defined as follows: for $\varphi_{h} \in \mathbb{R}_{r^{\prime}}\left(\widehat{\mathcal{T}}_{h}\right)$, and a Lagrange node $V \in \Omega$, $\mathcal{I}_{\text {av }}\left(\varphi_{h}\right)(V)=\left.\frac{1}{\left|\widehat{\mathcal{T}}_{V}\right|} \sum_{T \in \widehat{\mathcal{T}}_{V}} \varphi_{h}\right|_{T}(V)$, where $\widehat{\mathcal{T}}_{V}=\left\{T \in \widehat{\mathcal{T}}_{h}: V \in T\right\}$ and $|S|$ denotes the cardinality of a set $S$. Note that $\mathcal{I}_{\mathrm{av}}\left(\varphi_{h}\right)(V)=\varphi(V)$ at nodes $V$ lying in the interior of $T \in \widehat{\mathcal{T}}_{h}$. At boundary nodes, the value of $\mathcal{I}_{\text {av }}\left(\varphi_{h}\right)$ is set to zero. For the potential reconstruction, we set $s_{h}:=\mathcal{I}_{\text {av }}\left(\tilde{p}_{h}\right)$.

3.3.2. Construction of $\mathbf{t}_{h} \in \mathbf{V}_{\widehat{h}}$ by the solution of $h$-grid-size $k$-th order local Neumann problems. We define here a flux reconstruction $\mathbf{t}_{h}$ that is of lowerorder on the fine mesh $\widehat{\mathcal{T}}_{h}$, cf. Figure 2.2 (left); following [17]. We have $\mathbf{t}_{h} \in \mathbf{V}_{\widehat{h}}$ with $\nabla \cdot \mathbf{t}_{h}=P_{W_{\widehat{h}}}(f)$.

Consider those elements of $\mathcal{T}_{h}$ located in a band of width $H$ along the interface $\Gamma$ and regroup them into macro-elements $T$ of a macro-mesh $\mathcal{T}_{H}^{\Gamma}$. Denote the remaining elements of $\mathcal{T}_{h}$ by $\mathcal{T}_{H}^{\text {int }}$. We set

$$
\left.\mathbf{t}_{h}\right|_{T}:=\left.\mathbf{u}_{h}\right|_{T} \quad T \in \mathcal{T}_{H}^{\mathrm{int}} .
$$


For a macro-element $T \in \mathcal{T}_{H}^{\Gamma}$, we consider the mesh $\left.\widehat{\mathcal{T}}_{h}\right|_{T}$ and define on it the spaces $\mathbf{V}_{\widehat{h}, z, T}=\left\{\mathbf{v}_{h} \in \mathbf{V}_{\widehat{h}}(T): \mathbf{v}_{h} \cdot \mathbf{n}_{T}=z\right.$ on $\left.\partial T\right\}$, where $z$ is either $\left\{\left\{\mathbf{u}_{h} \cdot \mathbf{n}_{T}\right\}\right.$ or 0 . We seek $\left.\mathbf{t}_{h}\right|_{T} \in \mathbf{V}_{\widehat{h},\left\{\mathbf{u}_{h} \cdot \mathbf{n}_{T}\right\}, T}$ and $q_{h} \in W_{\widehat{h}}(T)$ satisfying

$$
\begin{aligned}
& \left(\mathbf{K}^{-1}\left(\mathbf{t}_{h}-\mathbf{u}_{h}\right), \mathbf{v}_{h}\right)_{T}-\left(q_{h}, \nabla \cdot \mathbf{v}_{h}\right)_{T}=0 \quad \forall \mathbf{v}_{h} \in \mathbf{V}_{\widehat{h}, 0, T}, \\
& \left(\nabla \cdot \mathbf{t}_{h}, w_{h}\right)_{T}=\left(f, w_{h}\right)_{T} \quad \forall w_{h} \in W_{\widehat{h}}(T) \text { such that }\left(w_{h}, 1\right)_{T}=0,
\end{aligned}
$$

with the additional condition $\left(q_{h}, 1\right)_{T}=0$. Let $\mathbf{t}_{h}^{\prime}:=\mathbf{t}_{h}-\mathbf{u}_{h}$. Then $\left(\mathbf{t}_{h}^{\prime}, q_{h}\right)$ corresponds to the $k$-th order mixed finite element approximation to the local Neumann problem on $T$

$$
\begin{aligned}
-\nabla \cdot(\mathbf{K} \nabla q) & =f-\nabla \cdot \mathbf{u}_{h} & & \text { in } T, \\
-\mathbf{K} \nabla q \cdot \mathbf{n}_{T} & =-\omega_{g} \llbracket \mathbf{u}_{h} \cdot \mathbf{n}_{g} \rrbracket & & \text { on all } g \in \mathcal{G}_{T}, \\
(q, 1)_{T} & =0, & &
\end{aligned}
$$

where $\omega_{g}:=\frac{1}{2}$ when $g \not \subset \partial \Omega$ and $\omega_{g}:=0$ when $g \subset \partial \Omega$. Note that these problems are well-posed as the Neumann boundary conditions are in equilibrium with the load,

$$
\sum_{g \in \mathcal{G}_{T}}\left\langle\mathbf{u}_{h} \cdot \mathbf{n}_{T}-\omega_{g} \llbracket \mathbf{u}_{h} \cdot \mathbf{n}_{g} \rrbracket, 1\right\rangle_{g}=\left\langle\left\{\left\{\mathbf{u}_{h} \cdot \mathbf{n}_{T}\right\}, 1\right\rangle_{\partial T}=(f, 1)_{T} .\right.
$$

The above follows by (3.14), Green's theorem, and Assumption 3.7 (2). For $\mathbf{t}_{h}$ satisfying (3.16), the Neumann boundary condition given by $\left\{\left\{\mathbf{u}_{h} \cdot \mathbf{n}_{T}\right\}\right.$ yields continuity of the normal component of $\mathbf{t}_{h}$ on $\partial T \cap \Gamma$, while (3.15) guarantees the same on $\partial T \backslash \Gamma$. Hence, $\mathbf{t}_{h} \in \mathbf{V}_{\widehat{h}}$. Finally, as the mixed finite element method minimizes the complementary energy, an equivalent way to rewrite (3.16) is

$$
\left.\mathbf{t}_{h}\right|_{T}=\arg \inf _{\mathbf{v}_{h} \in \mathbf{V}_{\widehat{h},\left\{\mathbf{u}_{h} \cdot \mathbf{n}_{T} \sharp, T\right.}, \nabla \cdot \mathbf{v}_{h}=P_{W_{\widehat{h}}}(f)}\left\|\mid \mathbf{u}_{h}-\mathbf{v}_{h}\right\|_{*, T} .
$$

Thus, on each $T \in \mathcal{T}_{H}^{\Gamma}, \mathbf{t}_{h}$ is the best choice from the space $\mathbf{V}_{\widehat{h},\left\{\mathbf{u}_{h} \cdot \mathbf{n}_{T}\right\}, T}$ to minimize the quantity $\left\|\mathbf{u}_{h}-\mathbf{v}_{h}\right\|_{*, T}$, subject to the constraint $\nabla \cdot \mathbf{v}_{h}=P_{W_{\hat{h}}}(f)$. Clearly, this is related to the definition of the $\eta_{\mathrm{M}, T}$ estimator, see (3.4).

REMARK 3.9 ( $\mathbf{t}_{h}$ given by (3.15)-(3.16)). The construction (3.15)-(3.16) is moderately expensive as mixed finite element approximations of order $k$ defined over $\mathrm{H}$ sized subdomains with h-sized grids need to be solved. We obtain local conservation on the fine mesh $\widehat{\mathcal{T}}_{h}$ and (3.5) takes the data oscillation form on $\widehat{\mathcal{T}}_{h}$. The mortar error is evaluated in the $H$-distance from the interface $\Gamma$, which, as we will see in Theorem 4.4, leads to its overestimation when $h \ll H$.

3.3.3. Construction of $\mathbf{t}_{h} \in \mathbf{V}_{H}$ by the solution of $H$-grid-size $m$-th order local Neumann problems. We define here $\mathbf{t}_{h}$ that is of higher-order on the coarse mesh $\mathcal{T}_{H}$, cf. Figure 2.2 (right), $\mathbf{t}_{h} \in \mathbf{V}_{H}$ with $\nabla \cdot \mathbf{t}_{h}=P_{W_{H}}(f)$.

In this section, we extend the mesh $\mathcal{G}_{H}$ from the interface $\Gamma$ to $\Gamma \cup \partial \Omega$ and refer to this extension as $\widetilde{G}_{H} ; \widetilde{G}_{H}$ is defined on $\partial \Omega$ by the sides of $\mathcal{T}_{H}$ lying on $\partial \Omega$. We also consider an extension of the mortar space $M_{H}, \widetilde{M}_{H}$, defined over $\widetilde{G}_{H}$ with the same $m$-th order piecewise discontinuous polynomials. Let the flux function $F$ be given by (3.13) on $\Gamma$. We extend it to $\Gamma \cup \partial \Omega$ by setting $\left.\widetilde{F}\right|_{\partial \Omega}:=P_{M_{H}}\left(\left.\left(\mathbf{u}_{h} \cdot \mathbf{n}_{\Omega}\right)\right|_{\partial \Omega}\right),\left.\widetilde{F}\right|_{\Gamma}=F$. For simplicity of notation, whenever we discuss the construction in Section 3.3.3, we will use $\mathcal{G}_{H}, M_{H}, F$ instead of $\widetilde{G}_{H}, \widetilde{M}_{H}, \widetilde{F}$, respectively.

Consider a fixed $\Omega_{i}$ and the mesh $\mathcal{T}_{H, i}$. We solve local Neumann problems by means of a $m$-th order mixed finite element method. Define the spaces

$$
\mathbf{V}_{H, Z, \Omega_{i}}=\left\{\mathbf{v}_{H} \in \mathbf{V}_{H}\left(\Omega_{i}\right): \mathbf{v}_{H} \cdot \mathbf{n}_{\Gamma}=Z \text { on } \Gamma_{i}, \mathbf{v}_{H} \cdot \mathbf{n}_{\Omega_{i}}=Z \text { on } \partial \Omega_{i} \cap \partial \Omega\right\},
$$


where $Z$ is either $F$ or 0 . We seek $\left.\mathbf{t}_{h}\right|_{\Omega_{i}} \in \mathbf{V}_{H, F, \Omega_{i}}$ and $q_{H} \in W_{H}\left(\Omega_{i}\right)$ satisfying

$$
\begin{aligned}
& \left(\mathbf{K}^{-1}\left(\mathbf{t}_{h}-\mathbf{u}_{h}\right), \mathbf{v}_{H}\right)_{\Omega_{i}}-\left(q_{H}, \nabla \cdot \mathbf{v}_{H}\right)_{\Omega_{i}}=0 \quad \forall \mathbf{v}_{H} \in \mathbf{V}_{H, 0, \Omega_{i}}, \\
& \left(\nabla \cdot \mathbf{t}_{h}, w_{H}\right)_{\Omega_{i}}=\left(f, w_{H}\right)_{\Omega_{i}} \quad \forall w_{H} \in W_{H}\left(\Omega_{i}\right) \text { such that }\left(w_{H}, 1\right)_{\Omega_{i}}=0,
\end{aligned}
$$

with the additional condition $\left(q_{H}, 1\right)_{\Omega_{i}}=0$. Once again, these problems are wellposed as

$$
\left\langle F \mathbf{n}_{\Omega_{i}} \cdot \mathbf{n}_{\Gamma}, 1\right\rangle_{\partial \Omega_{i} \cap \Gamma}+\langle F, 1\rangle_{\partial \Omega_{i} \cap \partial \Omega}=(f, 1)_{\Omega_{i}},
$$

by the same argument as in the previous section. We also have $\mathbf{t}_{h} \in \mathbf{V}_{H}$, as the Neumann boundary condition on $\Gamma$ given by $F$ yields continuity of the normal component of $\mathbf{t}_{h}$. Finally, as above, (3.18) is equivalent to

$$
\left.\mathbf{t}_{h}\right|_{\Omega_{i}}=\arg \inf _{\mathbf{v}_{H} \in \mathbf{V}_{H, F, \Omega_{i}}, \nabla \cdot \mathbf{v}_{H}=P_{W_{H}}(f)}|| \mathbf{u}_{h}-\mathbf{v}_{H} \mid \|_{*, \Omega_{i}} .
$$

REMARK 3.10 ( $\mathbf{t}_{h}$ given by (3.18)). The construction (3.18) is more expensive with mixed finite element approximations of order $m$ defined over the subdomains $\Omega_{i}$ with $H$-sized grids. We obtain local conservation on the mesh $\mathcal{T}_{H}$ and (3.5) takes the data oscillation form on $\mathcal{T}_{H}$. The mortar error is evaluated in the entire domain, which, as we will see in Theorem 4.4, leads to its optimal estimation in the multiscale setting when $h \ll H$.

4. Local efficiency of the a posteriori estimates. We derive here local efficiency of the estimators of Theorems 3.1 and 3.3. In order to proceed generally without the definition of any particular scheme, we rely on Assumptions 3.7 and 3.8. For convenience we will denote by $\eta \cdot, T$ the restrictions of the different estimators to each element $T$.

The following result is immediate using the triangle inequality:

Theorem 4.1 (Local efficiency of the diffusive flux and potential estimators). Let $(\mathbf{u}, p)$ satisfy (1.2). Let $\tilde{p}_{h} \in H^{1}\left(\mathcal{T}_{h}\right), \mathbf{u}_{h} \in \mathbf{L}^{2}(\Omega), s_{h} \in H_{0}^{1}(\Omega)$, and $\mathbf{t}_{h} \in \mathbf{H}(\operatorname{div}, \Omega)$ be arbitrary. Then, for all $T \in \mathcal{T}_{h}$,

$$
\begin{aligned}
\eta_{\mathrm{DF}, T} & \leq\left|\left\|\mathbf{u}-\mathbf{u}_{h}\left|\left\|_{*, T}+\right\|\right| p-\tilde{p}_{h}\right\|_{T},\right. \\
\eta_{\mathrm{P}, T} & \leq \eta_{\mathrm{DF}, T}+\eta_{\mathrm{NC}, T}, \\
\eta_{\mathrm{DFM}, T} & \leq \eta_{\mathrm{DF}, T}+\eta_{\mathrm{M}, T} .
\end{aligned}
$$

We will henceforth assume for simplicity that $f$ is a piecewise polynomial of degree $q$. We also assume that the mesh families $\left\{\mathcal{T}_{h}\right\}_{h>0},\left\{\widehat{\mathcal{T}}_{h}\right\}_{h>0}$, and $\left\{\mathcal{T}_{H}\right\}_{H>0}$ are shape-regular. A family $\left\{\mathcal{T}_{h}\right\}_{h>0}$ is shape-regular when there exists a constant $\kappa_{\mathcal{T}_{h}}>0$ such that $\min _{T \in \mathcal{T}_{h}} \rho_{T} / h_{T} \geq \kappa_{\mathcal{T}_{h}}$ for all $h>0$, where $\rho_{T}$ denotes the diameter of the largest ball inscribed in $T$. Note that shape-regularity does not exclude sharp local refinements. We also suppose that there exists a positive constant $C_{\mathcal{G}_{h}^{*}}$ such that, for all $g \in \mathcal{G}_{h}^{*}$,

$$
\frac{h_{g}}{h_{e}} \leq C_{\mathcal{G}_{h}^{*}} \quad \forall e \in \mathcal{E}_{h}^{\Gamma}, e \subset g .
$$

Assuming (4.1), we avoid the case where $h_{g} / h_{e}$ is only bounded by a function of $H / h$.

We will in the sequel use the notation $A \lesssim B$ to denote that $A \leq C B$. Here the constant $C$ depends on the space dimension $d$, the polynomial degrees $r$ of $\tilde{p}_{h}, r^{\prime}$ of $s_{h}, k$ of $\mathbf{u}_{h}, k$, or $m$ of $\mathbf{t}_{h}$, and $q$ of $f$, on the shape regularity parameters $\kappa_{\mathcal{T}_{h}}$ of $\mathcal{T}_{h}$, 
$\kappa_{\widehat{\mathcal{T}}_{h}}$ of $\widehat{\mathcal{T}}_{h}$, and $\kappa_{\mathcal{T}_{H}}$ of $\mathcal{T}_{H}$, on $\mathbf{K}$, and on the constant $C_{\mathcal{G}_{h}^{*}}$ from (4.1). However, $C$ is independent of any mesh size, the domain $\Omega$, and the regularity of the weak solution $(\mathbf{u}, p)$ of $(1.2)$.

For $T \in \mathcal{T}_{h}$, denote by $g_{T}$ all the sides in $\mathcal{G}_{h}^{*}$ that contain a node of $T$, and let $\mathfrak{T}_{T}=\left\{T^{\prime} \in \mathcal{T}_{h}: T^{\prime}\right.$ shares a node with $\left.T\right\}$ and $\mathfrak{T}_{T, \Gamma}=\left\{T^{\prime} \in \mathcal{T}_{h}: T^{\prime}\right.$ shares a node with $T$ or $\left.g_{T}\right\}$. Similarly, for a macro-element $T \in \mathcal{T}_{H}^{\Gamma}$, denote by $g_{T}$ all the sides in $\mathcal{G}_{H}$ which contains a side of $T$ and let $\mathfrak{T}_{T, \Gamma}=\left\{T^{\prime} \in \mathcal{T}_{h}: T^{\prime}\right.$ shares a node with $\left.g_{T}\right\}$. Set $h_{\mathfrak{T}_{T, \Gamma}}:=\min _{T^{\prime} \in \mathfrak{T}_{T, \Gamma}} h_{T^{\prime}}$.

We now establish the following two local efficiency theorems.

THEOREM 4.2 (Local efficiency of the nonconformity estimator). Let $p$ satisfy (1.1). Let Assumption 3.8 holds for $\tilde{p}_{h}$ and let $s_{h}$ be given by $s_{h}:=\mathcal{I}_{\text {av }}\left(\tilde{p}_{h}\right)$. Then, for all $T \in \mathcal{T}_{h}$,

$$
\begin{aligned}
& \eta_{\mathrm{NC}, T}\left.\lesssim\left\|p-\tilde{p}_{h}\right\|\right|_{\mathfrak{T}_{T}} \quad \text { if } T \cap \Gamma=\emptyset, \\
& \eta_{\mathrm{NC}, T} \lesssim\left\|p-\tilde{p}_{h}\right\| \|_{\mathfrak{T}_{T, \Gamma}} \quad \text { if } T \cap \Gamma \neq \emptyset .
\end{aligned}
$$

Proof. Let $T^{\prime} \in \widehat{\mathcal{T}}_{h}$. We proceed as in $[32,33]$, using the following two results: From $[21]$ we have, for any $\tilde{p}_{h} \in \mathbb{R}_{r^{\prime}}\left(\widehat{\mathcal{T}}_{h}\right)$,

$$
\left\|\nabla\left(\tilde{p}_{h}-\mathcal{I}_{\mathrm{av}}\left(\tilde{p}_{h}\right)\right)\right\|_{T^{\prime}} \leq C \sum_{e^{\prime} \in \widehat{\mathcal{E}}_{h} ; e^{\prime} \cap T^{\prime} \neq \emptyset} h_{e^{\prime}}^{-\frac{1}{2}}\left\|\llbracket \tilde{p}_{h} \rrbracket\right\|_{e^{\prime}},
$$

where $C$ depends only on $d, r^{\prime}$, and $\kappa_{\widehat{\mathcal{T}}_{h}}$. In [2, Theorem 10] it was established that for $e^{\prime} \in \widehat{\mathcal{E}}_{h}$ and $\tilde{p}_{h} \in H^{1}\left(\widehat{\mathcal{T}}_{h}\right)$ with $\left\langle\llbracket \tilde{p}_{h} \rrbracket, 1\right\rangle_{e^{\prime}}=0$,

$$
h_{e^{\prime}}^{-\frac{1}{2}}\left\|\llbracket \tilde{p}_{h} \rrbracket\right\|_{e^{\prime}} \leq C \sum_{T^{\prime} \in \widehat{\mathcal{T}}_{h} ; e^{\prime} \in \mathcal{E}_{T^{\prime}}}\left\|\nabla\left(\tilde{p}_{h}-\psi\right)\right\|_{T^{\prime}},
$$

where $\psi \in H_{0}^{1}(\Omega)$ is arbitrary and $C$ depends only on $d$ and $\kappa_{\widehat{\mathcal{T}}_{h}}$.

Let $T \in \mathcal{T}_{h}$ be such that $T \cap \Gamma=\emptyset$. Using the definition of $\widehat{\mathcal{T}}_{h}$, there exists an element $T^{\prime} \in \widehat{\mathcal{T}}_{h}$ such that $T=T^{\prime}$. Moreover, every side $e^{\prime} \in \widehat{\mathcal{E}}_{h}$ such that $e^{\prime} \cap T^{\prime} \neq \emptyset$ either coincides with a side $e \in \mathcal{E}_{h}^{\text {int }}$ or belongs to the interior of some $T^{\prime \prime} \in \mathcal{T}_{h}$ by assumption. Recalling Assumption 3.8 (1) and (2), we see that on each such side $e^{\prime},\left\langle\llbracket \tilde{p}_{h} \rrbracket, 1\right\rangle_{e^{\prime}}=0$. Combining (4.3) and (4.4) with $\psi=p,\left\|\nabla\left(\tilde{p}_{h}-\mathcal{I}_{\text {av }}\left(\tilde{p}_{h}\right)\right)\right\|_{T} \lesssim$ $\left\|\nabla\left(\tilde{p}_{h}-p\right)\right\|_{\mathfrak{T}_{T}}$, where we have used that $\widehat{\mathcal{T}}_{h}$ is a refinement of $\mathcal{T}_{h}$. Thus, (4.2a) follows after an appropriate scaling with respect to the tensor $\mathbf{K}$.

Now let $T \in \mathcal{T}_{h}$ such that $T \cap \Gamma \neq \emptyset$ be given. Using (4.3), we get

$$
\left\|\nabla\left(\tilde{p}_{h}-\mathcal{I}_{\mathrm{av}}\left(\tilde{p}_{h}\right)\right)\right\|_{T}^{2}=\sum_{T^{\prime} \in \widehat{\mathcal{T}}_{h} ; T^{\prime} \subset T}\left\|\nabla\left(\tilde{p}_{h}-\mathcal{I}_{\mathrm{av}}\left(\tilde{p}_{h}\right)\right)\right\|_{T^{\prime}}^{2} \leq C \sum_{e \in \widehat{\mathcal{E}}_{h} ; e \cap T \neq \emptyset} h_{e}^{-1}\left\|\llbracket \tilde{p}_{h}\right\|_{e}^{2} .
$$

For all $e \in \widehat{\mathcal{E}}_{h}$ such that $e \cap T \neq \emptyset$ and such that $e \not \subset \Gamma$, we have $\left\langle\llbracket \tilde{p}_{h} \rrbracket, 1\right\rangle_{e}=0$ by the same reasoning as above. Thus we can apply (4.4). All other sides $e$ are included in some $g \in \mathcal{G}_{h}^{*}$. We now use assumption (4.1) in combination with the assumption that $\widehat{\mathcal{T}}_{h}$ does not add any new nodes on $\Gamma$ with respect to $\mathcal{T}_{h}$. We conclude that

$$
\sum_{e \in \widehat{\mathcal{E}}_{h} ; e \subset g \in \mathcal{G}_{h}^{*}, g \cap T \neq \emptyset} h_{e}^{-1}\left\|\llbracket \tilde{p}_{h} \rrbracket\right\|_{e}^{2} \leq C_{\mathcal{G}_{h}^{*}} \sum_{g \in \mathcal{G}_{h}^{*}, g \cap T \neq \emptyset} h_{g}^{-1}\left\|\llbracket \tilde{p}_{h} \rrbracket\right\|_{g}^{2} .
$$

Using Lemma A.1 with $\psi=p,(4.2 \mathrm{~b})$ follows after an appropriate scaling with respect to the tensor $\mathbf{K}$.

REMARK 4.3 (Efficiency of $\eta_{\mathrm{NC}}$ ). We show in Section 5.1 that the multiscale mortar mixed finite element method of [8] satisfies Assumptions 3.8 (1)-(3). If a method 
only satisfies Assumption 3.8 (1), then one can establish $\eta_{\mathrm{NC}, T} \lesssim \sum_{e \in \widehat{\mathcal{E}}_{h} ; e \cap T \neq \emptyset} h_{e}^{-\frac{1}{2}}$ $\left\|\llbracket \tilde{p}_{h} \rrbracket\right\|_{e}$. Then, noticing that $\llbracket \tilde{p}_{h} \rrbracket=\llbracket \tilde{p}_{h}-p \rrbracket$ for all $e \in \widehat{\mathcal{E}}_{h}$, one can add $\sum_{e \in \widehat{\mathcal{E}}_{h}} h_{e}^{-\frac{1}{2}}$ $\left\|\llbracket \tilde{p}_{h} \rrbracket\right\|_{e}$ to both the error and estimate, as is usually done in the discontinuous Galerkin method, in order to obtain two-sided bounds in this case.

THEOREM 4.4 (Local efficiency of the residual and mortar estimators). Let $\mathbf{u}$ satisfy (1.2) and let Assumption 3.7 hold. We consider the following two cases:

Case 1) Let $\mathbf{t}_{h}$ be constructed as in Section 3.3.2. Then

$$
\begin{gathered}
\eta_{\mathrm{R}, \widehat{h}, T} \lesssim\left\|\mathbf{u}-\mathbf{u}_{h}\right\|_{*, T}, \quad T \in \widehat{\mathcal{T}}_{h}, \\
\eta_{\mathrm{M}, T}=0, \quad T \in \mathcal{T}_{H}^{\text {int }} .
\end{gathered}
$$

Let $T \in \mathcal{T}_{H}^{\Gamma}$ be a macro-element. In addition, suppose that $f \in \mathbb{R}_{k}\left(\mathcal{T}_{h}\right)$ and that $\nabla \cdot \mathbf{u}_{h}=P_{W_{h}}(f)$. Then

$$
\eta_{\mathrm{M}, T} \lesssim \sqrt{\frac{H_{T}}{h_{\mathfrak{T}_{T, \Gamma}}}}\left|\left\|\mathbf{u}-\mathbf{u}_{h}\right\|\right|_{*, \mathfrak{T}_{T, \Gamma}} .
$$

Case 2) Let $\mathbf{t}_{h}$ be constructed as in Section 3.3.3.

Assume sufficient smoothness leading to (4.11) below. Let $T \in \mathcal{T}_{H}$ be a coarse element and let $i \in\{1, \ldots, n\}$. Then

$$
\begin{aligned}
\eta_{\mathrm{R}, H, T} & \lesssim\left(\eta_{\mathrm{M}, T}+\mid\left\|\mathbf{u}-\mathbf{u}_{h}\right\|_{T}\right), \\
\eta_{\mathrm{M}, \Omega_{i}} & \leq \mid\left\|\mathbf{u}_{h}-\mathbf{u}\right\|_{*, \Omega_{i}}+\eta_{\mathrm{R}, h, \Omega_{i}}+C H^{m+1} .
\end{aligned}
$$

Proof. 1) We first prove (4.5)-(4.7), with $\mathbf{t}_{h}$ defined as in Section 3.3.2.

Using the element bubble function technique, cf. [31, Lemma 7.6], (4.5) is immediate. Next, (4.6) follows readily from (3.15). To prove (4.7), we fix a macro-element $T \in \mathcal{T}_{H}^{\Gamma}$ and let $\left(\mathbf{t}_{h}^{\prime}, q_{h}\right)$ be the solution of (3.17). We will denote by $T^{\prime}$ a generic element of $\left.\widehat{\mathcal{T}}_{h}\right|_{T}$. Consider the local postprocessing of $[6,31]$, see Section 5.1 below for more details. This gives $\tilde{q}_{h} \in \tilde{W}_{\widehat{h}}(T)$ such that $P_{\tilde{\mathbf{V}}_{\widehat{h}}}\left(-\mathbf{K} \nabla \tilde{q}_{h}\right)=\mathbf{t}_{h}^{\prime}$ and $P_{W_{\widehat{h}}}\left(\tilde{q}_{h}\right)=q_{h}$. Moreover, $\llbracket \tilde{q}_{h} \rrbracket$ is orthogonal to $\mathbb{R}_{k}(e)$ for any interior side $e$ of $\left.\widehat{\mathcal{T}}_{h}\right|_{T}$. Thus,

$$
\begin{aligned}
\left\|\mathbf{t}_{h}^{\prime}\right\|_{*, T}^{2} & =\left(\mathbf{K}^{-1} \mathbf{t}_{h}^{\prime}, \mathbf{t}_{h}^{\prime}\right)_{T}=-\left(\nabla \tilde{q}_{h}, \mathbf{t}_{h}^{\prime}\right)_{T} \\
& =\sum_{T^{\prime} \subset T}\left\{\left(\tilde{q}_{h}, \nabla \cdot \mathbf{t}_{h}^{\prime}\right)_{T^{\prime}}-\left\langle\tilde{q}_{h}, \mathbf{t}_{h}^{\prime} \cdot \mathbf{n}_{T^{\prime}}\right\rangle_{\partial T^{\prime}}\right\}=\left\langle\tilde{q}_{h}, \frac{1}{2} \llbracket \mathbf{u}_{h} \cdot \mathbf{n}_{g} \rrbracket\right\rangle_{\partial T \cap \Gamma} \\
& =\left\langle\tilde{q}_{h}-\left(\tilde{q}_{h}\right)_{T}, \frac{1}{2} \llbracket \mathbf{u}_{h} \cdot \mathbf{n}_{g} \rrbracket\right\rangle_{\partial T \cap \Gamma} \\
& \leq C \sum_{g \in \mathcal{G}_{H}, g \subset \partial T}\left\|\llbracket \mathbf{u}_{h} \cdot \mathbf{n}_{g} \rrbracket\right\|_{g} H_{T}^{\frac{1}{2}} c_{\mathbf{K}, T}^{-\frac{1}{2}}\|\| \tilde{q}_{h}\|\|_{T} \\
& \leq C\left\|\mathbf{t}_{h}^{\prime}\right\|_{*, T} \sum_{g \in \mathcal{G}_{H}, g \subset \partial T}\left\|\llbracket \mathbf{u}_{h} \cdot \mathbf{n}_{g} \rrbracket\right\|_{g} H_{T}^{\frac{1}{2}} c_{\mathbf{K}, T}^{-\frac{1}{2}} .
\end{aligned}
$$

Here we have used the definition of the postprocessing in (4.9a) and Green's theorem in $(4.9 \mathrm{~b})$. The observations that $\nabla \cdot \mathbf{t}_{h}^{\prime}=P_{W_{\widehat{h}}}\left(f-\nabla \cdot \mathbf{u}_{h}\right)=0$ since $f-\nabla \cdot \mathbf{u}_{h}=0$ and that $\tilde{q}_{h} \in \tilde{W}_{\widehat{h}}(T)$ as well as $\mathbf{t}_{h}^{\prime} \cdot \mathbf{n}_{T}=0$ on $\partial T \backslash \Gamma$ and $\mathbf{t}_{h}^{\prime} \cdot \mathbf{n}_{T}=-\frac{1}{2} \llbracket \mathbf{u}_{h} \cdot \mathbf{n}_{g} \rrbracket$ on all 
$g \subset \partial T \cap \Gamma$ yield the second equality in (4.9b). Applying $\left(\tilde{q}_{h}, 1\right)_{T}=0$ which follows from the assumption $\left(q_{h}, 1\right)_{T}=0$ and from $P_{W_{\hat{h}}}\left(\tilde{q}_{h}\right)=q_{h}$ results in (4.9c). The discrete trace inequality $\left\|\tilde{q}_{h}-\left(\tilde{q}_{h}\right)_{T}\right\|_{g} \leq C H_{T}^{\frac{1}{2}}\left\|\nabla \tilde{q}_{h}\right\|_{T}$, which can be obtained as discrete Poincaré's and Friedrichs' inequalities in [30, Theorems 5.4 and 8.1] gives (4.9d). Finally (4.9e) is obtained using the inequality (cf. [33, Lemma 5.4]) $\left\|\tilde{q}_{h}\right\|\left\|_{T} \leq C\right\|\left\|\mathbf{t}_{h}^{\prime}\right\|_{*, T}$. Thus $\eta_{\mathrm{M}, T}=\left\|\left|\mathbf{t}_{h}-\mathbf{u}_{h}\right|\right\|_{*, T}=\left\|\mid \mathbf{t}_{h}^{\prime}\right\|_{*, T}$ is bounded by the terms $\left\|\llbracket \mathbf{u}_{h} \cdot \mathbf{n}_{g} \rrbracket\right\|_{g} H_{T}^{\frac{1}{2}}$, which are estimated by (4.7) in [35].

2) We now prove (4.8), with $\mathbf{t}_{h}$ defined as in Section 3.3.3.

Let $T \in \mathcal{T}_{H}$ be given. We first note that using the element bubble function technique and the triangle inequality,

$$
C_{\mathrm{P}, T} H_{T} c_{\mathbf{K}, T}^{-\frac{1}{2}}\left\|f-\nabla \cdot \mathbf{t}_{h}\right\|_{T} \leq C C_{\mathbf{K}, T}^{\frac{1}{2}} c_{\mathbf{K}, T}^{-\frac{1}{2}}\left(\left\|\mathbf{u}_{h}-\mathbf{t}_{h}\right\|\left\|_{*, T}+\right\| \mathbf{u}-\mathbf{u}_{h} \|_{*, T}\right) .
$$

Next let $i \in\{1, \ldots, n\}$ and consider the subdomain problem:

$$
\begin{aligned}
-\nabla \cdot(\mathbf{K} \nabla q) & =f & & \text { in } \Omega_{i}, \\
-\mathbf{K} \nabla q \cdot \mathbf{n}_{\Omega_{i}} & =\left.F\right|_{\partial \Omega_{i}}\left(\mathbf{n}_{\Omega_{i}} \cdot \mathbf{n}_{\Gamma}\right) & & \text { on } \partial \Omega_{i}, \\
(q, 1)_{\Omega_{i}} & =(p, 1)_{\Omega, i} . & &
\end{aligned}
$$

Denote by $\left(\mathbf{t}_{H}^{\#}, q_{H}^{\#}\right)$ its mixed finite element approximation in $\mathbf{V}_{H, F, \Omega_{i}} \times W_{H}\left(\Omega_{i}\right)$. Using (3.19) we obtain

$$
\left\|\mathbf{u}_{h}-\mathbf{t}_{h}\right\|\left\|_{*, \Omega_{i}} \leq\right\| \mathbf{u}_{h}-\mathbf{t}_{H}^{\#} \mid\left\|_{*, \Omega_{i}} \leq\right\| \mathbf{t}_{H}^{\#}+\mathbf{K} \nabla q\left\|_{*, \Omega_{i}}+\right\| \mathbf{u}_{h}+\mathbf{K} \nabla q \|_{*, \Omega_{i}} .
$$

Assuming that $q$ is sufficiently smooth, we have

$$
\left\|\mathbf{t}_{H}^{\#}+\mathbf{K} \nabla q\right\|_{*, \Omega_{i}} \leq C H^{m+1} .
$$

Using [33, Theorem 3.1] yields

$$
\left\|\mathbf{u}_{h}+\mathbf{K} \nabla q\right\|\left\|_{*, \Omega_{i}} \leq\right\| \mathbf{u}_{h}+\mathbf{K} \nabla p\|\|_{*, \Omega_{i}}+\left|\left(\mathbf{u}_{h}+\mathbf{K} \nabla q, \frac{\nabla(p-q)}{\|p-q\| \|_{\Omega_{i}}}\right)_{\Omega_{i}}\right| .
$$

Setting $\varphi:=(p-q) /\|p-q\| \|_{\Omega_{i}}$ and noting that $\varphi \in H^{1}\left(\Omega_{i}\right)$ with $\varphi_{\Omega_{i}}=0$ we have

$$
\begin{aligned}
& \left\|\left|\mathbf{u}_{h}+\mathbf{K} \nabla q\|\|_{*, \Omega_{i}} \leq\right|\left|\mathbf{u}_{h}-\mathbf{u}\|\|_{*, \Omega_{i}}+\right|\left(\mathbf{u}_{h}+\mathbf{K} \nabla q, \nabla \varphi\right)_{\Omega_{i}} \mid\right. \\
= & \left\|\left|\mathbf{u}_{h}-\mathbf{u} \|_{*, \Omega_{i}}+\right|\left(f-\nabla \cdot \mathbf{u}_{h}, \varphi\right)_{\Omega_{i}}+\left\langle\left(\mathbf{u}_{h}+\mathbf{K} \nabla q\right) \cdot \mathbf{n}_{\Omega_{i}}, \varphi\right\rangle_{\partial \Omega_{i}} \mid\right. \\
= & \left\|\left|\mathbf{u}_{h}-\mathbf{u} \|_{*, \Omega_{i}}+\right|\left(f-\nabla \cdot \mathbf{u}_{h}, \varphi\right)_{\Omega_{i}}+\left\langle\mathbf{u}_{h} \cdot \mathbf{n}_{\Omega_{i}}-\left.F\right|_{\partial \Omega_{i}}\left(\mathbf{n}_{\Omega_{i}} \cdot \mathbf{n}_{\Gamma}\right), \varphi-P_{M_{H}}(\varphi)\right\rangle_{\partial \Omega_{i}} \mid\right. \\
\leq & \left\|\left|\mathbf{u}_{h}-\mathbf{u}\left\|_{*, \Omega_{i}}+\eta_{\mathrm{R}, h, \Omega_{i}}+\right\| \mathbf{u}_{h} \cdot \mathbf{n}_{\Omega_{i}}-F\right|_{\partial \Omega_{i}}\left(\mathbf{n}_{\Omega_{i}} \cdot \mathbf{n}_{\Gamma}\right)\right\|_{\partial \Omega_{i}}\left\|\varphi-P_{M_{H}}(\varphi)\right\|_{\partial \Omega_{i}} .
\end{aligned}
$$

The first term in the above inequality is the actual error; the second one is generally of higher order and can be bounded as in (4.5). In addition, if both $p$ and $q$ (and consequently $\varphi$ ) are sufficiently smooth, from $[8$, estimate (3.5)] we have

$$
\left\|\varphi-P_{M_{H}}(\varphi)\right\|_{\partial \Omega_{i}} \leq C H^{m+1} .
$$

Both estimates (4.11) and (4.12) are, of course, not sharp as $C$ is unknown constant and depends on the smoothness of $p$ and $q$. We expect that the term $\| \mathbf{u}_{h} \cdot \mathbf{n}_{\Omega_{i}}$ $\left.F\right|_{\partial \Omega_{i}}\left(\mathbf{n}_{\Omega_{i}} \cdot \mathbf{n}_{\Gamma}\right) \|_{\partial \Omega_{i}}$ to be bounded in view of (3.13).

REMARK 4.5 (Robustness with respect to the ratio $H / h$ ). Theorems 4.1 and 4.2 (cf. also Remark 4.3) give the overestimation factor independent of the ratio $\mathrm{H} / \mathrm{h}$. 
According to Theorem 4.4, the flux reconstruction of Section 3.3.2 does not lead to a similar multiscale robustness result. A different situation appears to arise using the reconstruction of Section 3.3.3. The a priori error estimates presented in [8] for the multiscale mortar mixed finite element method indicate that in that case, $\left\|\mathbf{u}_{h}-\mathbf{u}\right\| \|_{*}$ converges as $\mathcal{O}\left(H^{m+\frac{1}{2}}\right)$, so that the factor $C H^{m+1}$ of $(4.8 \mathrm{~b})$ is of higher order. This multiscale robustness is not optimal as it hinges on the high regularity of the exact solution and a priori arguments, but is demonstrated numerically in Section 7 below.

5. Multiscale, multinumerics, and mortar discretizations. We present here different methods that fit into the framework of the previous sections. In order to check that the presented a posteriori error estimates and their efficiencies hold true, we only need to verify that Assumptions 3.7 and 3.8 (1) or 3.8 (1)-(3) are satisfied.

5.1. Multiscale mortar mixed finite element method. The multiscale mortar mixed finite element method [8,7] for (1.2) is defined as: Find $\mathbf{u}_{h} \in \mathbf{V}_{h}, p_{h} \in W_{h}$, and $\lambda_{H} \in M_{H}$ such that,

$$
\begin{array}{ll}
\left(\mathbf{K}^{-1} \mathbf{u}_{h}, \mathbf{v}_{h}\right)_{\Omega_{i}}-\left(p_{h}, \nabla \cdot \mathbf{v}_{h}\right)_{\Omega_{i}}+\left\langle\lambda_{H}, \mathbf{v}_{h} \cdot \mathbf{n}_{\Omega_{i}}\right\rangle_{\Gamma_{i}}=0 & \forall \mathbf{v}_{h} \in \mathbf{V}_{h, i}, \forall i, \\
\left(\nabla \cdot \mathbf{u}_{h}, w_{h}\right)_{\Omega_{i}}=\left(f, w_{h}\right)_{\Omega_{i}} & \forall w_{h} \in W_{h, i}, \forall i, \\
\sum_{i=1}^{n}\left\langle\mathbf{u}_{h} \cdot \mathbf{n}_{\Omega_{i}}, \mu_{H}\right\rangle_{\Gamma_{i}}=0 & \forall \mu_{H} \in M_{H} .
\end{array}
$$

Here $\mathbf{u}_{h}$ immediately satisfies Assumption 3.7. The $p_{h}$ obtained from (5.1) is not suitable to be used as $\tilde{p}_{h}$ in the a posteriori framework of Sections $3-4$, see the discussion in $[31,33]$. We devote the rest of this section to obtaining a suitable $\tilde{p}_{h}$.

Let $i$ be fixed and let $\Lambda_{h, i}$ be the usual Lagrange multiplier space associated with $\mathbf{V}_{h, i} \times W_{h, i}$, see $[12,29]$. Let $\tilde{\mathbf{V}}_{h, i}$ be the space without the interelement constraints, $\tilde{\mathbf{V}}_{h, i}:=\bigoplus_{T \in \mathcal{T}_{h, i}} \mathbf{V}_{h, i}(T)$. Set $\Lambda_{h}:=\bigoplus_{i=1}^{n} \Lambda_{h, i}, \tilde{\mathbf{V}}_{h}:=\bigoplus_{i=1}^{n} \tilde{\mathbf{V}}_{h, i}$. Let $\left(\mathbf{u}_{h}, p_{h}\right)$ be the solution of (5.1). We define $\lambda_{h} \in \Lambda_{h}$ by

$$
\left\langle\lambda_{h}, \mathbf{v}_{e} \cdot \mathbf{n}_{T}\right\rangle_{e}:=-\left(\mathbf{K}^{-1} \mathbf{u}_{h}, \mathbf{v}_{e}\right)_{T}+\left(p_{h}, \nabla \cdot \mathbf{v}_{e}\right)_{T}
$$

for all flux basis functions $\mathbf{v}_{e}$ of $\tilde{\mathbf{V}}_{h, i}$ associated with the element $T \in \mathcal{T}_{h}$ and its side $e$. Let $P_{\tilde{\mathbf{V}}_{h}}$ be the $\mathbf{L}^{2}(\Omega)$-orthogonal projection onto $\tilde{\mathbf{V}}_{h}$ with respect to the scalar product $\left(\mathbf{K}^{-1} \cdot, \cdot\right)$ and $P_{\Lambda_{h}}$ the $L^{2}\left(\mathcal{E}_{h}^{\text {int, }, \Gamma}\right)$-orthogonal projection onto $\Lambda_{h}$, i.e.,

$$
\begin{array}{llll}
P_{\tilde{\mathbf{V}}_{h}}: \mathbf{L}^{2}(\Omega) \rightarrow \tilde{\mathbf{V}}_{h} & \text { for } \mathbf{v} \in \mathbf{L}^{2}(\Omega), & \left(\mathbf{K}^{-1}\left(\mathbf{v}-P_{\tilde{\mathbf{V}}_{h}}(\mathbf{v})\right), \mathbf{v}_{h}\right)=0 & \forall \mathbf{v}_{h} \in \tilde{\mathbf{V}}_{h}, \\
P_{\Lambda_{h}}: L^{2}\left(\mathcal{E}_{h}^{\text {int, }, \Gamma}\right) \rightarrow \Lambda_{h} & \text { for } \mu \in L^{2}\left(\mathcal{E}_{h}^{\text {int, }, \Gamma}\right), & \left(\mu-P_{\Lambda_{h}}(\mu), \mu_{h}\right)_{\mathcal{E}_{h}^{\text {int, }, \Gamma}}=0 & \forall \mu_{h} \in \Lambda_{h} .
\end{array}
$$

Our basic tool for the a posteriori error analysis of the mixed finite element method will be the local postprocessing of the potential $p_{h}$ introduced in [6], see also [31]. Let $\tilde{W}_{h}$ be a polynomial space of functions $\varphi_{h}$ satisfying

$$
\left\langle\llbracket \varphi_{h} \rrbracket, \psi_{h}\right\rangle_{e}=0 \quad \forall e \in \mathcal{E}_{h}^{\text {int }} \cup \mathcal{E}_{h}^{\text {ext }}, \forall \psi_{h} \in \mathbb{R}_{k}(e)
$$

and specified in $[6,31]$. Then we define:

Definition 5.1 (Postprocessing $\tilde{p}_{h}$ of $p_{h}$ ). We define $\tilde{p}_{h} \in \tilde{W}_{h}$ by

$$
\begin{aligned}
P_{W_{h}}\left(\tilde{p}_{h}\right) & =p_{h}, \\
P_{\Lambda_{h}}\left(\tilde{p}_{h}\right) & =\lambda_{h} .
\end{aligned}
$$


Note that employing (5.4) in (5.2) and using $\nabla \cdot \mathbf{V}_{h}(T)=W_{h}(T)$ and $\left.\mathbf{V}_{h}(T) \cdot \mathbf{n}_{T}\right|_{\partial T \backslash \partial \Omega}=\Lambda_{h}(T)$ gives, for all $T \in \mathcal{T}_{h}$,

$$
\left(\mathbf{K}^{-1} \mathbf{u}_{h}, \mathbf{v}_{h}\right)_{T}-\left(\tilde{p}_{h}, \nabla \cdot \mathbf{v}_{h}\right)_{T}+\left\langle\tilde{p}_{h}, \mathbf{v}_{h} \cdot \mathbf{n}_{T}\right\rangle_{\partial T \backslash \partial \Omega}=0 \quad \forall \mathbf{v}_{h} \in \mathbf{V}_{h}(T) .
$$

Applying Green's theorem for the two last terms in the above expression gives

$$
\left(\mathbf{K}^{-1}\left(\mathbf{u}_{h}+\mathbf{K} \nabla \tilde{p}_{h}\right), \mathbf{v}_{h}\right)_{T}=0 \quad \forall \mathbf{v}_{h} \in \mathbf{V}_{h}(T) \quad \forall T \in \mathcal{T}_{h},
$$

which implies $P_{\tilde{\mathbf{V}}_{h}}\left(-\mathbf{K} \nabla \tilde{p}_{h}\right)=\mathbf{u}_{h}$. We refer to [6,33] for more details.

The postprocessed potential $\tilde{p}_{h}$ satisfies Assumption $3.8(1)$ as $\tilde{W}_{h}$ is a piecewise polynomial space. Moreover, Assumption 3.8 (2) is implied by (5.3). The following lemma shows that Assumption 3.8 (3) for the above $\tilde{p}_{h}$ holds as well.

Lemma 5.2 (Weak continuity of $\tilde{p}_{h}$ ). For any union of interface sides $g \in \mathcal{G}_{h}^{*}$,

$$
\left\langle\llbracket \tilde{p}_{h} \rrbracket, \psi_{h}\right\rangle_{g}=0 \quad \forall \psi_{h} \in \mathbb{R}_{k}(g) .
$$

Moreover, for all $e \in \mathcal{E}_{h}^{\Gamma}$, let $T_{e}$ be the element of $\mathcal{T}_{h}$ having e as side. Then

$$
\left\langle\left.\tilde{p}_{h}\right|_{T_{e}}-\lambda_{H}, \psi_{h}\right\rangle_{e}=0 \quad \forall \psi_{h} \in \mathbb{R}_{k}(e) .
$$

Proof. Fix $e \in \mathcal{E}_{h}^{\Gamma}$ and take all basis functions $\mathbf{v}_{e}$ in (5.1a). Using (5.5) and (5.4a) yields $\left\langle-\left.\tilde{p}_{h}\right|_{T_{e}}+\lambda_{H}, \mathbf{v}_{e} \cdot \mathbf{n}_{T_{e}}\right\rangle_{e}=0$; whence (5.7) follows. To prove (5.6), take any $g \in \mathcal{G}_{h}^{*}$. Thus $g$ is given by one or more sides from some $\Omega_{i}$ and by one or more sides from some $\Omega_{j}$. By summing (5.7) over sides, we obtain (5.6).

5.2. Multiscale mortar discontinuous Galerkin method. We consider here the multiscale mortar discontinuous Galerkin (DG) method of [19] for the problem (1.1). For simplicity, let $\mathbf{K}$ be piecewise constant on $\mathcal{T}_{h}$ in this section and let $k \geq 1$. Then the method reads: Find $p_{h} \in W_{h}$ and $\lambda_{H} \in M_{H}$ such that

$\sum_{i=1}^{n} \sum_{g \in \mathcal{G}_{H, i}}\left\langle-\left.\mathbf{K} \nabla p_{h}\right|_{\Omega_{i}} \cdot \mathbf{n}_{\Omega_{i}}+\alpha_{g} \frac{\sigma_{\mathbf{K}, g}}{H_{g}}\left(\left.p_{h}\right|_{\Omega_{i}}-\pi_{k, \mathcal{E}_{h, i}^{\Gamma}}\left(\lambda_{H}\right)\right), \mu_{H}\right\rangle_{g}=0 \quad \forall \mu_{H} \in M_{H}$,

where $\mathcal{B}_{h, i}\left(p_{h}, \lambda_{H} ; \varphi_{h}\right)$ is given by

$$
\begin{aligned}
& -\sum_{e \in \mathcal{E}_{h, i}^{\text {int }}}\left\{\left\langle\left\{\mathbf{K} \nabla p_{h} \cdot \mathbf{n}_{e}\right\}, \llbracket \varphi_{h} \rrbracket\right\rangle_{e}+\theta\left\langle\left\{\left\{\mathbf{K} \nabla \varphi_{h} \cdot \mathbf{n}_{e}\right\}, \llbracket p_{h} \rrbracket\right\rangle_{e}+\left\langle\alpha_{e} \frac{\sigma_{\mathbf{K}, e}}{h_{e}} \llbracket p_{h} \rrbracket, \llbracket \varphi_{h} \rrbracket\right\rangle_{e}\right\}\right. \\
& -\sum_{g \in \mathcal{G}_{H, i}}\left\{\left\langle\left.\mathbf{K} \nabla p_{h}\right|_{\Omega_{i}} \cdot \mathbf{n}_{\Omega_{i}}-\alpha_{g} \frac{\sigma_{\mathbf{K}, g}}{H_{g}}\left(\left.p_{h}\right|_{\Omega_{i}}-\lambda_{H}\right),\left.\varphi_{h}\right|_{\Omega_{i}}\right\rangle_{g}\right. \\
& \left.+\bar{\theta}\left\langle\left.\mathbf{K} \nabla \varphi_{h}\right|_{\Omega_{i}} \cdot \mathbf{n}_{\Omega_{i}},\left.p_{h}\right|_{\Omega_{i}}-\lambda_{H}\right\rangle_{g}\right\}+\left(\mathbf{K} \nabla p_{h}, \nabla \varphi_{h}\right)_{\Omega_{i}} .
\end{aligned}
$$

Here $\alpha_{e}, e \in \mathcal{E}_{h}^{\text {int }}$, and $\alpha_{g}, g \in \mathcal{G}_{H}$, are the penalty parameters (taken sufficiently large), $\sigma_{\mathbf{K}, e}$ and $\sigma_{\mathbf{K}, g}$ are $\mathbf{K}$-dependent weights, and $\theta, \bar{\theta} \in\{-1,0,1\}$ lead to the usual choices of the various discontinuous Galerkin schemes. For the sake of simplicity, we suppose here that $\mathcal{E}_{h, i}^{\Gamma}$ is a refinement of $\mathcal{G}_{H, i}$ for all $i \in\{1, \ldots, n\}$. In fact, in comparison with [19], we have replaced in $(5.8 \mathrm{~b}) \lambda_{H}$ by $\pi_{k, \mathcal{E}_{h, i}^{\Gamma}}\left(\lambda_{H}\right)$. Note that as 
$\varphi_{h} \in W_{h, i},\left.\varphi_{h}\right|_{\Gamma_{i}}$ is a piecewise polynomial of order $k$ on $\mathcal{E}_{h, i}^{\Gamma}$. Consequently, we can equivalently replace $\lambda_{H}$ by $\pi_{k, \mathcal{E}_{h, i}^{\Gamma}}\left(\lambda_{H}\right)$ also in the fifth term of (5.9). Likewise, it is equivalent to replace $\lambda_{H}$ by $\pi_{k, \mathcal{E}_{h, i}^{\Gamma}}\left(\lambda_{H}\right)$ in the multiscale mortar mixed finite element method (5.1).

Following $[22,16]$, we now introduce the flux $\mathbf{u}_{h}$. We will use it in our a posteriori error estimates but we remark that it can be of independent interest.

Definition 5.3 (DG flux reconstruction). Let $T \in \mathcal{T}_{h}$. Then the reconstructed flux $\left.\mathbf{u}_{h}\right|_{T} \in \mathbf{V}_{h}(T)$ is given by

$$
\begin{gathered}
\left\langle\mathbf{u}_{h} \cdot \mathbf{n}_{e}, q_{h}\right\rangle_{e}=\left\langle-\left\{\left[\mathbf{K} \nabla p_{h} \cdot \mathbf{n}_{e}\right\}\right]+\alpha_{e} \frac{\sigma_{\mathbf{K}, e}}{h_{e}} \llbracket p_{h} \rrbracket, q_{h}\right\rangle_{e} \\
\forall q_{h} \in \mathbb{R}_{k}(e), \forall e \in \mathcal{E}_{T}, e \not \subset \Gamma, \\
\left\langle\mathbf{u}_{h} \cdot \mathbf{n}_{e}, q_{h}\right\rangle_{e}=\left\langle-\mathbf{K} \nabla p_{h} \cdot \mathbf{n}_{e}+\alpha_{g} \frac{\sigma_{\mathbf{K}, g}}{H_{g}}\left(p_{h}-\lambda_{H}\right), q_{h}\right\rangle_{e} \\
\forall q_{h} \in \mathbb{R}_{k}(e), \forall e \in \mathcal{E}_{T}, e \subset g \in \mathcal{G}_{H},
\end{gathered}
$$

$$
\begin{gathered}
\left(\mathbf{u}_{h}, \mathbf{r}_{h}\right)_{T}=-\left(\mathbf{K} \nabla p_{h}, \mathbf{r}_{h}\right)_{T}+\theta \sum_{e \in \mathcal{E}_{T}, e \not \subset \Gamma} \omega_{e}\left\langle\mathbf{K} \mathbf{r}_{h} \cdot \mathbf{n}_{e}, \llbracket p_{h} \rrbracket\right\rangle_{e} \\
+\bar{\theta} \sum_{e \in \mathcal{E}_{T}, e \subset \Gamma}\left\langle\mathbf{K r}_{h} \cdot \mathbf{n}_{e},\left(p_{h}-\lambda_{H}\right) \mathbf{n}_{T} \cdot \mathbf{n}_{e}\right\rangle_{e} \\
\forall \mathbf{r}_{h} \in \mathbb{R}_{k-1, *, d}(T),
\end{gathered}
$$

where $\omega_{e}:=\frac{1}{2}$ if $e \in \mathcal{E}_{h}^{\text {int }}$ and $\omega_{e}:=1$ if $e \in \mathcal{E}_{h}^{\text {ext }}$.

We now establish the following

Lemma 5.4 (DG reconstructed flux property). Let $\mathbf{u}_{h}$ be given by Definition 5.3. Then $\mathbf{u}_{h}$ satisfies Assumption 3.7.

Proof. Assumption 3.7 (1) holds by construction (the normal components on sides from $\mathcal{E}_{h, i}^{\text {int }}$ are continuous). Let $i \in\{1, \ldots, n\}, T \in \mathcal{T}_{h, i}$, and $\xi_{h} \in \mathbb{R}_{k}(T)$ be arbitrary. Since $\left.\xi_{h}\right|_{e} \in \mathbb{R}_{k}(e)$ for all $e \in \mathcal{E}_{T}, \nabla \xi_{h} \in \mathbb{R}_{k-1, *, d}(T)$, and $\mathbf{u}_{h}$ satisfies (5.10), we have with $\varphi_{h}=\xi_{h}$ on $T$ and $\varphi_{h}=0$ otherwise,

$$
\left(\nabla \cdot \mathbf{u}_{h}, \xi_{h}\right)_{T}=-\left(\mathbf{u}_{h}, \nabla \xi_{h}\right)_{T}+\sum_{e \in \mathcal{E}_{T}}\left\langle\mathbf{u}_{h} \cdot \mathbf{n}_{T}, \xi_{h}\right\rangle_{e}=\mathcal{B}_{h, i}\left(p_{h}, \lambda_{H} ; \varphi_{h}\right)=\left(f, \xi_{h}\right)_{T}
$$

by Green's theorem. Thus, $\nabla \cdot \mathbf{u}_{h}=\pi_{k}(f)$, and, consequently Assumption 3.7 (2) follows. Finally, Assumption 3.7 (3) is immediate from (5.8b) noting that (5.10b) implies, on all $e \in \mathcal{E}_{h, i}^{\Gamma}$,

$$
\left.\mathbf{u}_{h}\right|_{\Omega_{i}} \cdot \mathbf{n}_{e}=-\left.\mathbf{K} \nabla p_{h}\right|_{\Omega_{i}} \cdot \mathbf{n}_{e}+\alpha_{g} \frac{\sigma_{\mathbf{K}, g}}{H_{g}}\left(\left.p_{h}\right|_{\Omega_{i}}-\pi_{k, \mathcal{E}_{h, i}^{\Gamma}}\left(\lambda_{H}\right)\right) .
$$

REMARK 5.5 (Potential $\tilde{p}_{h}$ in the multiscale mortar DG method). The solution $p_{h} \in W_{h}$ can directly be used as $\tilde{p}_{h}$. Such potential $\tilde{p}_{h}$ in general only satisfies Assumption 3.8 (1) and not Assumptions 3.8 (2)-(3).

5.3. Multiscale mortar coupled mixed finite element-discontinuous Galerkin method. We give here an example of a multinumerics discretization. Following [19], we consider the multiscale mortar coupled mixed finite element-discontinuous Galerkin method. For simplicity, we assume $\mathbf{K}$ is piecewise constant on $\mathcal{T}_{h}$. Let $I_{\mathrm{DG}}$ denote the index set of the subdomains where the $k$-th order DG method is used and $I_{\mathrm{MFE}}$ the index set of the subdomains where the $k$-th order mixed finite element 
method is used. We seek, for $k \geq 1,\left.p_{h}\right|_{\Omega_{i}} \in W_{h, i}, i \in I_{\mathrm{DG}},\left.\left(\mathbf{u}_{h}, p_{h}\right)\right|_{\Omega_{i}} \in \mathbf{V}_{h, i} \times W_{h, i}$, $i \in I_{\mathrm{MFE}}$, and $\lambda_{H} \in M_{H}$ such that

(5.11a) $\mathcal{B}_{h, i}\left(p_{h}, \lambda_{H} ; \varphi_{h}\right)=\left(f, \varphi_{h}\right)_{\Omega_{i}} \quad \forall \varphi_{h} \in W_{h, i}, i \in I_{\mathrm{DG}}$,

(5.11b) $\left(\mathbf{K}^{-1} \mathbf{u}_{h}, \mathbf{v}_{h}\right)_{\Omega_{i}}-\left(p_{h}, \nabla \cdot \mathbf{v}_{h}\right)_{\Omega_{i}}+\left\langle\lambda_{H}, \mathbf{v}_{h} \cdot \mathbf{n}_{\Omega_{i}}\right\rangle_{\Gamma_{i}}=0 \quad \forall \mathbf{v}_{h} \in \mathbf{V}_{h, i}, i \in I_{\mathrm{MFE}}$,

(5.11c) $\left(\nabla \cdot \mathbf{u}_{h}, w_{h}\right)_{\Omega_{i}}=\left(f, w_{h}\right)_{\Omega_{i}} \quad \forall w_{h} \in W_{h, i}, i \in I_{\mathrm{MFE}}$

$$
\begin{aligned}
& \sum_{i \in J}\left\langle\mathbf{u}_{h} \cdot \mathbf{n}_{\Omega_{i}}, \mu_{H}\right\rangle_{\Gamma_{i}}+\sum_{i \in I} \sum_{g \in \mathcal{G}_{H, i}}\left\langle-\left.\mathbf{K} \nabla p_{h}\right|_{\Omega_{i}} \cdot \mathbf{n}_{\Omega_{i}}\right. \\
& \left.\quad+\alpha_{g} \frac{\sigma_{\mathbf{K}, g}}{H_{g}}\left(\left.p_{h}\right|_{\Omega_{i}}-\pi_{k, \mathcal{E}_{h, i}^{\Gamma}}\left(\lambda_{H}\right)\right), \mu_{H}\right\rangle_{g}=0 \quad \forall \mu_{H} \in M_{H} .
\end{aligned}
$$

The following lemma is a straightforward generalization of the above results:

Lemma 5.6 (Coupled mixed finite element-DG flux property). Let $\left.\mathbf{u}_{h}\right|_{\Omega_{i}} \in$ $\mathbf{V}_{h, i}, i \in I_{M F E}$, be given by (5.11). Define $\left.\mathbf{u}_{h}\right|_{\Omega_{i}} \in \mathbf{V}_{h, i}, i \in I_{D G}$, using the flux reconstruction of Definition 5.3. Then $\mathbf{u}_{h}$ satisfies Assumption 3.7.

Thus, defining $\left.\tilde{p}_{h}\right|_{\Omega_{i}}$ by $\left.p_{h}\right|_{\Omega_{i}}$ for $i \in I_{\mathrm{DG}}$ and by the postprocessing (5.4) for $i \in I_{\mathrm{MFE}}$, the results of Sections 3-4 can be applied to the coupling (5.11).

5.4. Multiscale mortar finite volume methods. The present approach can be easily extended to finite volume-type multiscale mortar methods and their couplings with other multiscale mortar methods, following the example of Section 5.3 in combination with the results of [32].

6. An a posteriori error estimate without flux reconstruction. We describe here briefly an alternative a posteriori error estimate not requiring any construction of a globally $\mathbf{H}(\operatorname{div}, \Omega)$-conforming flux $\mathbf{t}_{h}$.

For a given subdomain $\Omega_{i}$ and a given interface side $g \in \mathcal{G}_{H, i}$, let $T_{i, g}$ denote the element of a mesh $\mathcal{T}_{H, i}$ having $g$ as a side. Here, $\mathcal{T}_{H}$ is a coarse-scale mesh described in Section 2.1. Recall the trace inequality $\left\|\varphi-\varphi_{g}\right\|_{g} \leq C_{\mathrm{t}, T_{i, g}, g} h_{g}^{\frac{1}{2}}\|\nabla \varphi\|_{T_{i, g}}$. It has been shown in [24, Lemma 3.5] that $C_{\mathrm{t}, T_{i, g}, g}=\left(C_{\mathrm{t}, d}\right)^{\frac{1}{2}}\left(|g| h_{T_{i, g}}^{2} /\left(\left|T_{i, g}\right| h_{g}\right)\right)^{\frac{1}{2}}$, where $C_{\mathrm{t}, d} \approx$ 0.77708 for a triangle, $C_{\mathrm{t}, d} \approx 3.84519$ for a tetrahedron, and $C_{\mathrm{t}, d}=1 /(\pi \tanh \pi)$ for a rectangle. Then it holds (see [26] for the proof):

Corollary 6.1 (Alternative estimate for the flux and the potential). Let $\mathbf{u}$ be the exact flux defined by (1.2), let $p$ be the exact potential defined by (1.1), let $\mathbf{u}_{h}$ satisfy Assumption 3.7, and let $\tilde{p}_{h} \in H^{1}\left(\mathcal{T}_{h}\right)$ be arbitrary. Let $s_{h} \in H_{0}^{1}(\Omega)$. Then

$$
\begin{aligned}
\left\|\mathbf{u}-\mathbf{u}_{h}\right\|_{*} & \leq \eta_{\mathrm{P}}+\eta_{\mathrm{R}, h}+\tilde{\eta}_{\mathrm{M}}, \\
\left\|p-\tilde{p}_{h}\right\| & \leq \eta_{\mathrm{NC}}+\eta_{\mathrm{R}, h}+\tilde{\eta}_{\mathrm{M}}+\eta_{\mathrm{DF}},
\end{aligned}
$$

with $\eta_{\mathrm{P}}$ given by (3.3), $\eta_{\mathrm{NC}}$ by (3.9), $\eta_{\mathrm{DF}}$ by $(3.11), \eta_{\mathrm{R}, h}$ by (3.5) with $\mathcal{T}_{\hbar}=\mathcal{T}_{h}$, and

$$
\tilde{\eta}_{\mathrm{M}}:=\left\{\sum_{i=1}^{n} \sum_{j=1}^{n} \sum_{g \in \mathcal{G}_{H, i, j}}\left(\frac{1}{2}\left\|\llbracket \mathbf{u}_{h} \cdot \mathbf{n}_{g} \rrbracket\right\|_{g} C_{\mathrm{t}, T_{i, g}, g} H_{g}^{\frac{1}{2}} c_{\mathbf{K}, T_{i, g}}^{-\frac{1}{2}}\right)^{2}\right\}^{\frac{1}{2}} .
$$

It follows from $[35,26]$ that the efficiency of $\tilde{\eta}_{\mathrm{M}}$ is as that in Theorem 4.4, case 1.

7. Numerical experiments. We present here several numerical experiments for problem (1.1) illustrating the different a posteriori error estimators.

We will use the following shorthand notation:

- Method 1: Estimates of Theorems 3.1 and 3.3, flux reconstruction of Section 3.3.2. 

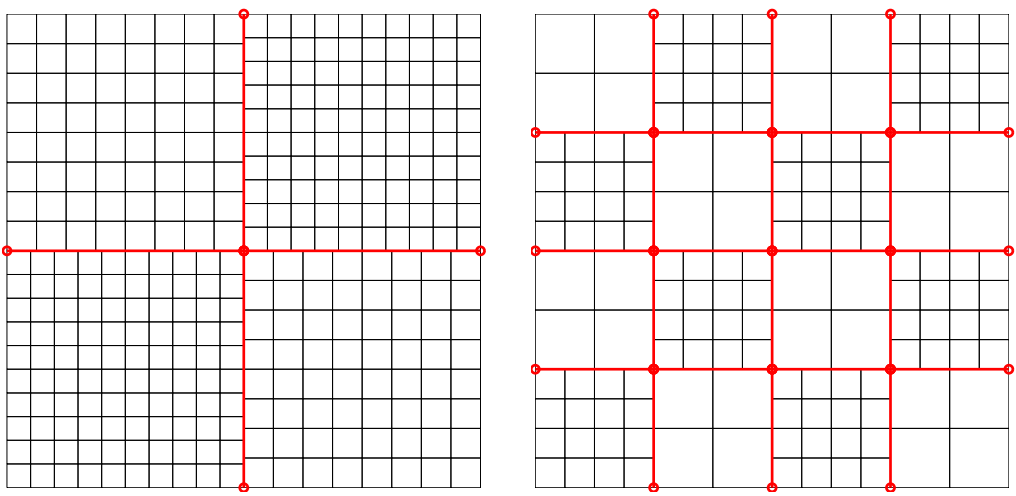
(right)

FIG. 7.1. Initial subdomains grid $\mathcal{T}_{h}$ and interface grid $\mathcal{G}_{H}$, Section 7.1 (left) and Section 7.2

- Method 2: Estimates of Theorems 3.1 and 3.3, flux reconstruction of Section 3.3.3.

- Method 3: Estimates of Corollary 6.1, no flux reconstruction needed.

Since the paper focuses on three major issues, mortars, multiscale, and multinumerics, we consider three test examples, each emphasizing one of these issues. In each case we reduce the problem to a coarse scale interface operator and use the multiscale mortar basis method developed in $[18,34]$ to solve the coarse scale interface equations.

7.1. Mortar coupling. This first example focuses on the mortar coupling. We set $\Omega:=(0,1) \times(0,1)$ and take a diagonal highly oscillating tensor coefficient $\mathbf{K}$,

$$
\mathbf{K}:= \begin{cases}15-10 \sin (10 \pi x) \sin (10 \pi y), & x, y \in(0,1 / 2) \text { or } x, y \in(1 / 2,1), \\ 15-\sin (2 \pi x) \sin (2 \pi y), & \text { otherwise. }\end{cases}
$$

We impose the source term $f$ according to the analytic solution $p(x, y)=x(1-x)$ $y(1-y)$ and use the multiscale mortar mixed finite element method (5.1). The domain $\Omega$ is divided into four subdomains $\Omega_{i}$; in each subdomain $\Omega_{i}$, we use the lowestorder Raviart-Thomas-Nédélec mixed finite element method on a square mesh $\mathcal{T}_{h, i}$, $\mathbf{V}_{h, i}:=\operatorname{RTN}^{0}\left(\mathcal{T}_{h, i}\right), W_{h, i}:=\mathbb{R}_{0}\left(\mathcal{T}_{h, i}\right)$. Thus $k=0$. The mortar space $M_{H}$ is the space of discontinuous first-order polynomials on the interface mesh $\mathcal{G}_{H}$. Thus $m=1$. The meshes $\mathcal{T}_{h, i}$ do not match along the interface $\Gamma$, see Figure 7.1 (left). We perform several levels of uniform grid refinement where both subdomain and mortar element diameters are halved, so that $H=4 \sqrt{2} h$ on each level. Note that this setting is rather extreme, with coarse mortar grid and fine subdomain grids and relatively large $C_{\mathcal{G}_{h}^{*}}=5$. We have chosen this example to test our estimates when the mortar error is significant.

Figure 7.2 (left) compares the actual and estimated flux errors $\left\|\mathbf{u}-\mathbf{u}_{h}\right\| \|_{*}$ against the total number of degrees of freedom for Methods 1 and 3. The corresponding effectivity indices, given as the ratios of the estimate over the error, are plotted in Figure 7.2 (right). We see that the estimates give an upper bound on the error, as predicted by the theory. In Figure 7.3 (left), we plot the mortar estimators $\eta_{\mathrm{M}}$ for Method 1 and $\tilde{\eta}_{\mathrm{M}}$ for Method 3 . We can see that Method 1 exibits better results for both the mortar error and the overall error.

Figure 7.3 (right) compares the flux estimators $\eta_{\mathrm{P}}, \eta_{\mathrm{R}, \hbar}$, and $\eta_{\mathrm{M}}$ for Method 1. The estimator $\eta_{\mathrm{P}}$, as well as the flux error $\left\|\mathbf{u}-\mathbf{u}_{h}\right\|_{*}$, converges as $\mathcal{O}(h)$. Since $f$ is 

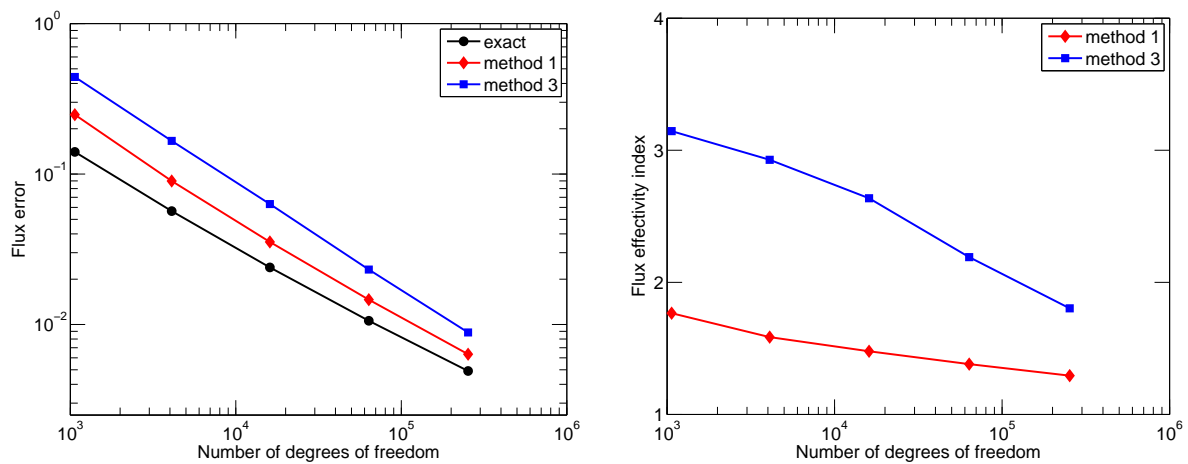

FIG. 7.2. Estimated and actual flux error (left) and effectivity indices (right) on uniformly refined meshes using Methods 1 and 3 for Section 7.1
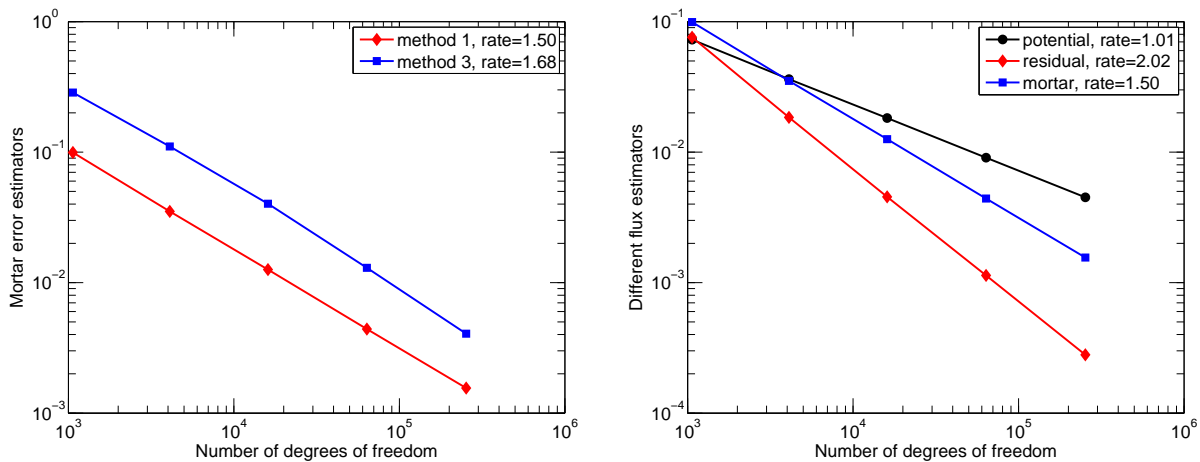

FIG. 7.3. Mortar estimators using Methods 1 and 3 (left) and different estimators using Method 1 (right) on uniformly refined meshes for Section 7.1

smooth, $\eta_{\mathrm{R}, \hbar}$ converges as $\mathcal{O}\left(h^{2}\right)$. Here, $\eta_{\mathrm{M}}$ converges faster than $\mathcal{O}(h)$ and eventually becomes negligible. For the same reason, the overall precision of both Methods 1 and 3 becomes closer with increasing refinement levels, cf. Figure 7.2.

We next show in Figure 7.4 the estimated and actual spatial distribution of the flux errors $\left\|\mathbf{u}-\mathbf{u}_{h}\right\|_{*}$ of Method 1 on the third level refinement. We can see that the error estimator can detect the actual error distribution very well. In particular, both the error inside each subdomain and the mortar error along the interface $\Gamma$ are well predicted.

7.2. Multiscale. The second example focuses on the multiscale setting and compares all Methods 1, 2, and 3. We set $\Omega:=(0,1) \times(0,1)$,

$$
\mathbf{K}:=\left(\begin{array}{ll}
3 & 2 \\
2 & 3
\end{array}\right),
$$

and impose $f$ according to the analytic solution $p(x, y)=\sin (2 \pi x) \sin (2 \pi y)$. Note that $p \in C^{\infty}(\Omega)$ and hence is smooth enough for the proof of Theorem 4.4, case 2.

As in the previous example, we use the multiscale mortar mixed finite element method (5.1). The subdomains $\Omega_{i}$, the initial subdomain meshes $\mathcal{T}_{h, i}$, and the mortar mesh $\mathcal{G}_{H}$ are illustrated in Figure 7.1 (right). We keep $\mathbf{V}_{h, i}:=\mathbf{R T N} \mathbf{N}^{0}\left(\mathcal{T}_{h, i}\right), W_{h, i}:=$ $\mathbb{R}_{0}\left(\mathcal{T}_{h, i}\right)$, i.e., $k=0$. We, however, increase the polynomial order approximation on 

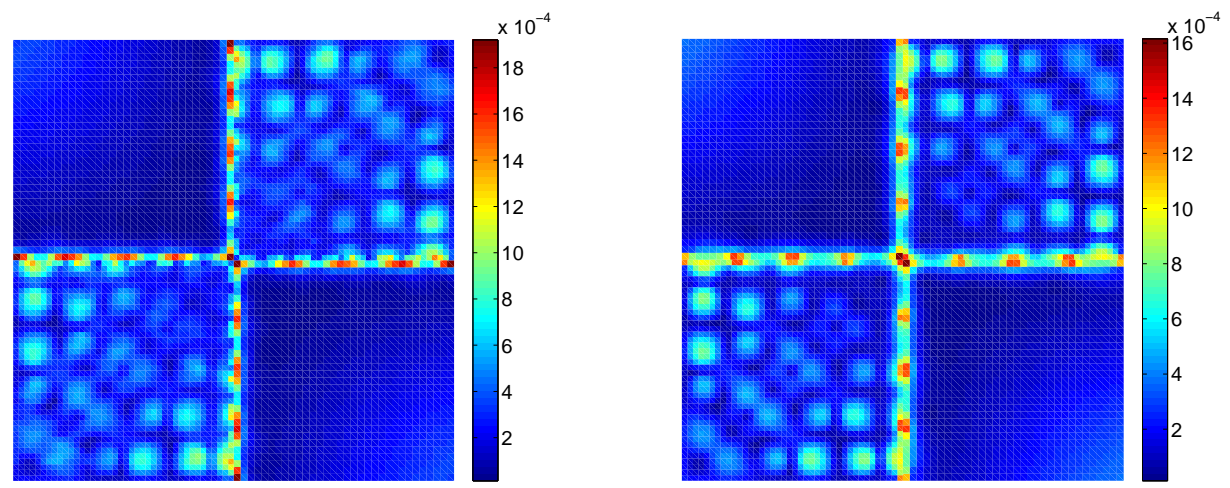

FIG. 7.4. Estimated (left) and actual (right) flux error distribution on a uniformly refined mesh using Method 1 for Section 7.1
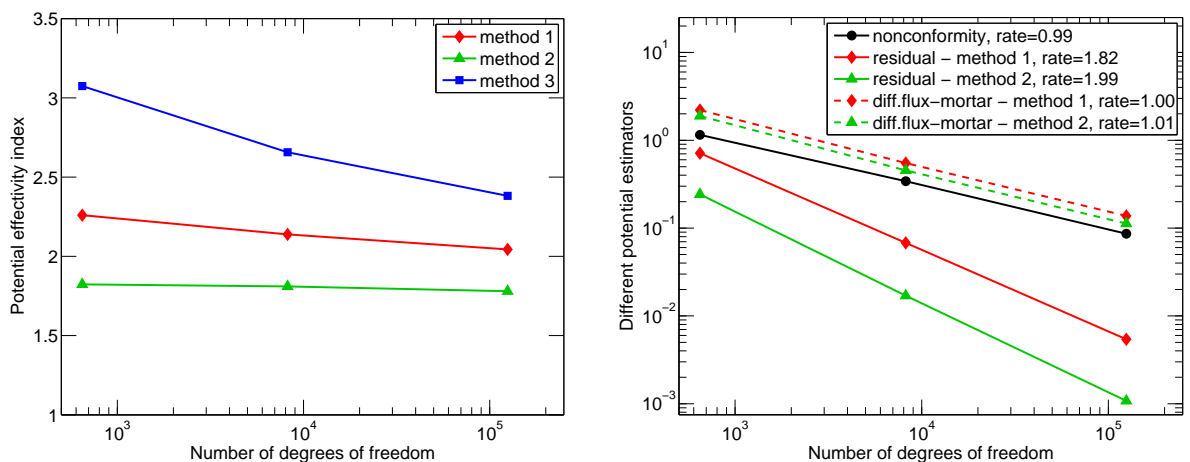

FIG. 7.5. Potential effectivity indices using methods 1, 2, and 3 (left) and different potential estimators using methods 1 and 2 (right) on uniformly refined meshes for Section 7.2, case (a)

the interface mesh $\mathcal{G}_{H}$. More precisely, we consider two cases, where $M_{H}$ consists of discontinuous piecewise quadratic polynomials, i.e., $m=2$, in case (a), and of discontinuous piecewise linear polynomials, i.e., $m=1$, in case (b). For both cases, we perform several levels of uniform grid refinement where we halve mortar element diameters and reduce four times the subdomain element diameters, so $H=2^{-\frac{3}{4}} \sqrt{h}$.

Case (a) corresponds to the a priori analysis of [8] and to our robustness result for method 2 in Remark 4.5. Case (b) is not covered by the a priori theory nor by Remark 4.5 but is much more computationally efficient than case (a). Recall that (5.1) can be reduced to an interface problem on $M_{H}$, which is given in case (b) only by piecewise first-order polynomials on $\mathcal{G}_{H}$. For both cases we observe the same accuracy; the overall error decreases as $\mathcal{O}(h)$. It should be noted that no relation between $k$ and $m$ is required for our estimates nor for their efficiency, except for method 2 in Theorem 4.4 and Remark 4.5. So we can use our estimates to monitor the asymptotic accuracy, especially in case (b), where the mortar error is expected to be rather significant and presents a challenging test.

Figure 7.5 shows the results for case (a) and Figure 7.6 for case (b). As predicted by Theorem 4.4, the estimators $\eta_{\mathrm{M}}$ of method 1 and $\tilde{\eta}_{\mathrm{M}}$ of method 3 are not robust with respect to the ratio $H / h$ (see in particular Figure 7.6 (right), where $\eta_{\mathrm{M}}$ decays with a slope $\mathcal{O}\left(h^{0.64}\right)$ inferior to $\mathcal{O}(h)$ of $\eta_{\mathrm{P}, T}$ and of $\left.\left\|\left|\mathbf{u}-\mathbf{u}_{h}\right|\right\|_{*}\right)$. Consequently, the 

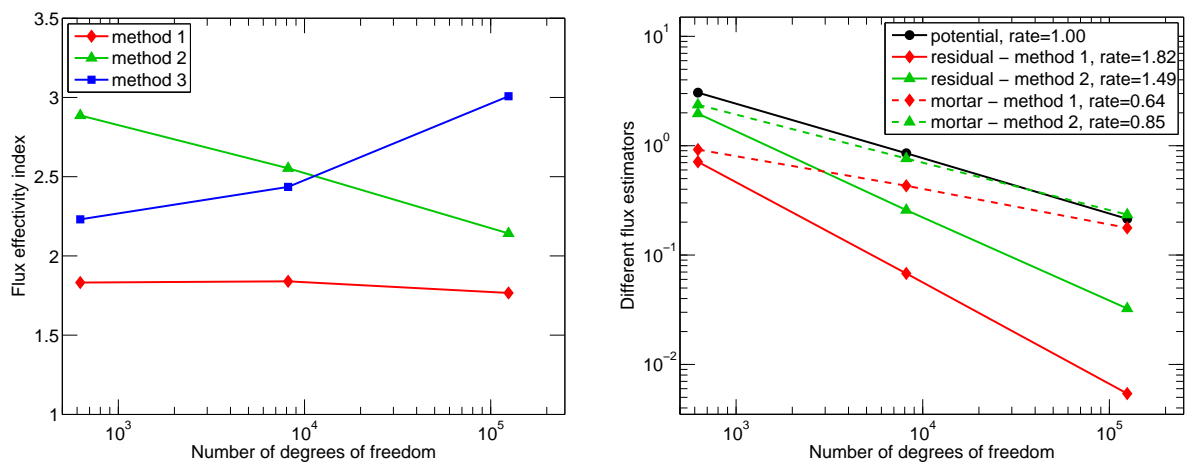

FIG. 7.6. Flux effectivity indices using methods 1, 2, and 3 (left) and different flux estimators using methods 1 and 2 (right) on uniformly refined meshes for Section 7.2, case (b)
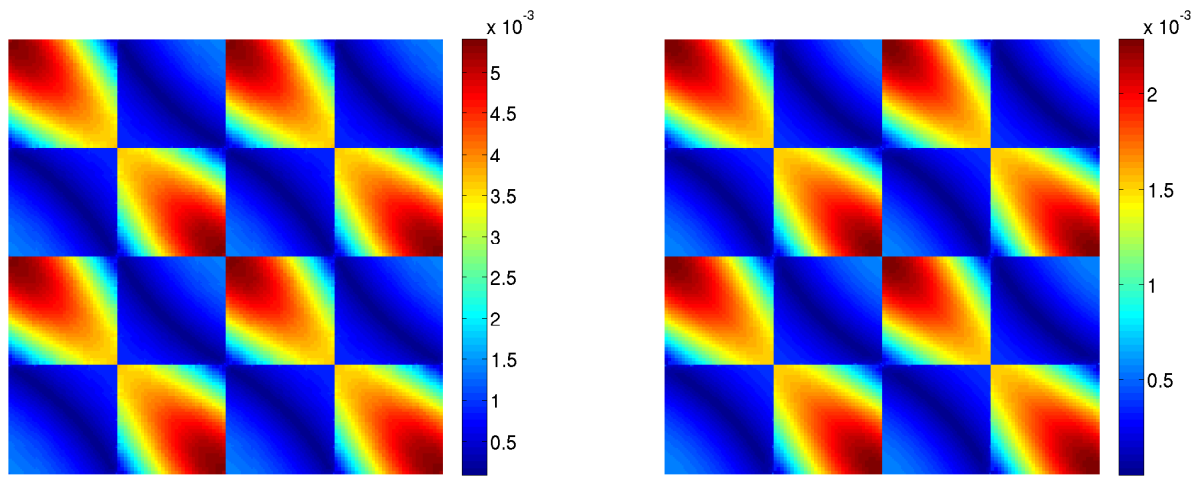

FIG. 7.7. Estimated (left) and actual (right) flux error distribution on a uniformly refined mesh using method 2 for Section 7.2, case (a)

effectivity index, see method 3 in Figure 7.6 (left), although quite close to the optimal value of one on coarse meshes, grows with the refinement level. On the other hand, as predicted by Theorem 4.4 in conjunction with Remark 4.5 , the estimator $\eta_{\mathrm{M}}$ of method 2 is fully robust with respect to the ratio $H / h$; see Figure 7.5 (right), where $\eta_{\mathrm{M}}$ for method 2 decays with the slope $\mathcal{O}(h)$ of $\eta_{\mathrm{P}, T}$ and of $\left\|\mathbf{u}-\mathbf{u}_{h} \mid\right\|_{*}$. The rate in Figure 7.6 (right) is no longer optimal, as not covered by Remark 4.5 (0.85 instead of 1), but is still much better than 0.64 of method 1. The estimated and the actual spatial distribution of the flux errors || $\mathbf{u}-\mathbf{u}_{h} \mid \|_{*}$ in case (a) for method 2 on the third level of refinement are shown in Figure 7.7. They once again match closely.

7.3. Multinumerics and adaptivity. The third example focuses on the multinumerics and local adaptivity of both the subdomain and mortar grids. Set $\Omega:=$ $(-1,1) \times(-1,1)$ and

$$
\mathbf{K}:= \begin{cases}5 & (x, y) \in(-1,0) \times(-1,0) \text { or }(x, y) \in(0,1) \times(0,1), \\ 1 & \text { otherwise }\end{cases}
$$

We impose the source term $f$ and Dirichlet boundary conditions according to the analytic solution, which is given (in polar coordinates) by $p(r, \theta) \mid \Omega_{i}=r^{\alpha}\left(a_{i} \sin (\alpha \theta)+\right.$ $\left.b_{i} \cos (\alpha \theta)\right)$, where $i \in\{1, \ldots, 4\}$ corresponds to the axis quadrants and where $\alpha=$ $0.53544, a_{1}=0.44721, b_{1}=1, a_{2}=-0.74536, b_{2}=2.33333, a_{3}=-0.94412, b_{3}=$ 

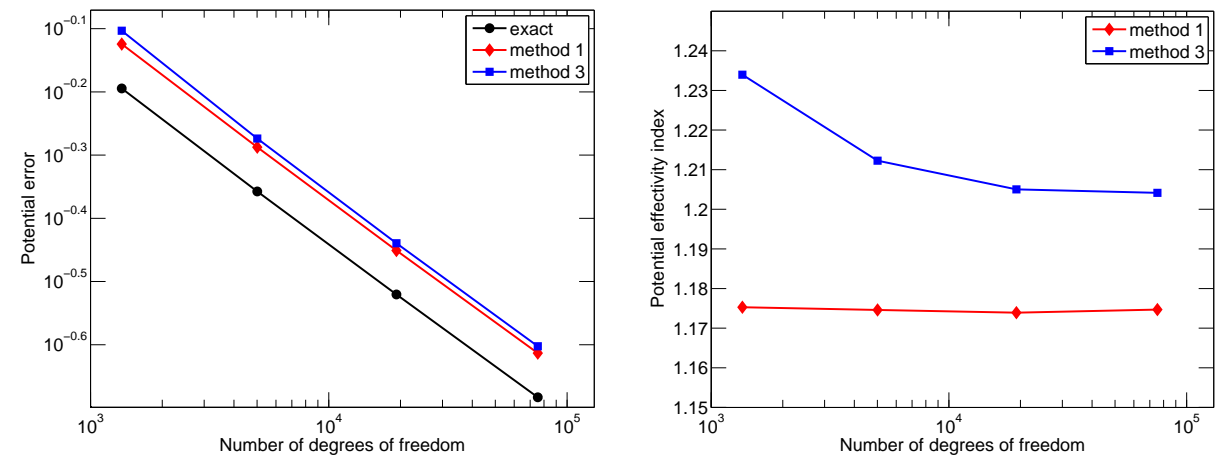

FIG. 7.8. Estimated and actual potential error (left) and effectivity indices (right) on uniformly refined meshes using methods 1 and 3 for Section 7.3

0.55556, $a_{4}=-2.4017$, and $b_{4}=-0.48148$. Inhomogeneous Dirichlet boundary conditions are set according to the solution; the error stemming from their discrete approximation is neglected. This solution has been studied previously in $[28,31,32]$ and provides an excellent test for a posteriori error estimation and adaptive mesh refinement due to the singularity at the point $(0,0)$.

The domain $\Omega$ is divided into sixteen subdomains $\Omega_{i}$ with the interface $\Gamma$ along the lines $x=-1 / 2,0,1 / 2$ and $y=-1 / 2,0,1 / 2$. On the inner subdomains, i.e., those which intersect the point $(0,0)$ where the singularity resides, we use piecewise linear Non-symmetric Interior Penalty Galerkin (NIPG) finite element method on triangular meshes [19]. In the remaining subdomains we use the lowest-order Raviart-ThomasNédélec mixed finite element method on a square mesh. The coupling is achieved via (5.11).

The mortar space $M_{H}$ is the space of discontinuous second-order polynomials on the interface mesh $\mathcal{G}_{H}(m=2)$. For initial meshes we use $4 \times 4$ rectangular meshes in the mixed subdomains and similar $4 \times 4$ meshes divided into triangles in the NIPG subdomains. The initial mortar grid $\mathcal{G}_{H}$ has one element on all $\mathcal{G}_{H, i, j}$, so that $H=1 / 4$ for the coarsest mesh. We note that the initial subdomain grids match along the interface, but in the adaptive algorithm the subdomains may each be refined independently.

Figure 7.8 (left) compares the actual and estimated potential errors for methods 1 and 3 on uniformly refined meshes. The corresponding effectivity indices are plotted in Figure 7.8 (right). We observe very good behavior in this multinumerics setting.

Next, we use the a posteriori error estimate for adaptive mesh refinement. For our refinement criteria, we compute the maximum of the subdomain and mortar error indicators and mark a subdomain or mortar mesh element for refinement if its error indicator is larger that 0.8 times this maximum. Within the mixed finite element subdomains the grids are refined uniformly, while within the NIPG subdomains and on the mortar interface grid the elements are refined locally.

Figure 7.9 shows the adapted subdomain (left) and mortar (right) mesh after twelve refinements using method 1 . We see that the refinements are concentrated around the singularity. Figure 7.10 (left) gives the corresponding effectivity indices.

Finally, in Figure 7.10 (right) we compare the estimated and actual flux errors on uniform and adaptive meshes. Clearly, the adaptively refined meshes are able to provide an accurate solution with far fewer degrees of freedom. The convergence 

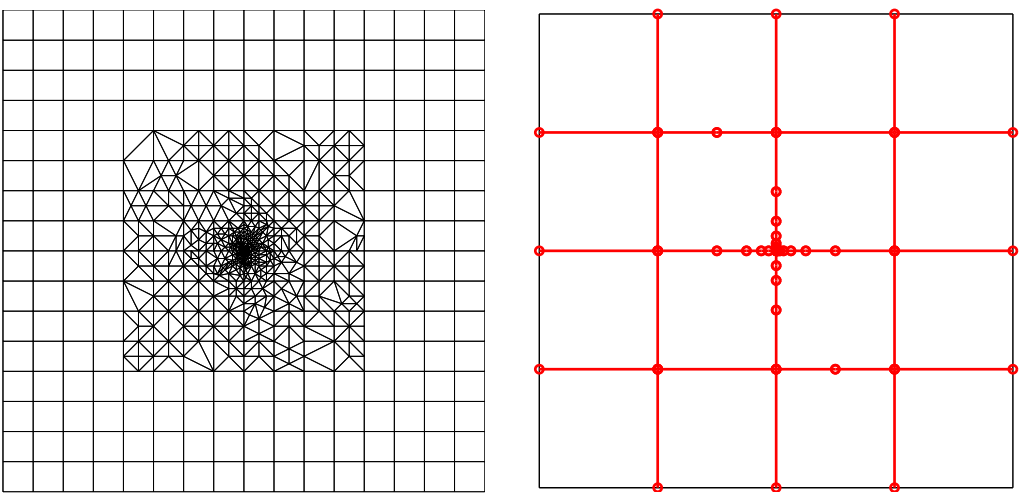

FIG. 7.9. Adaptive subdomain mesh (left) and mortar mesh (right) after 12 steps of adaptive refinement using method 1 for Section 7.3
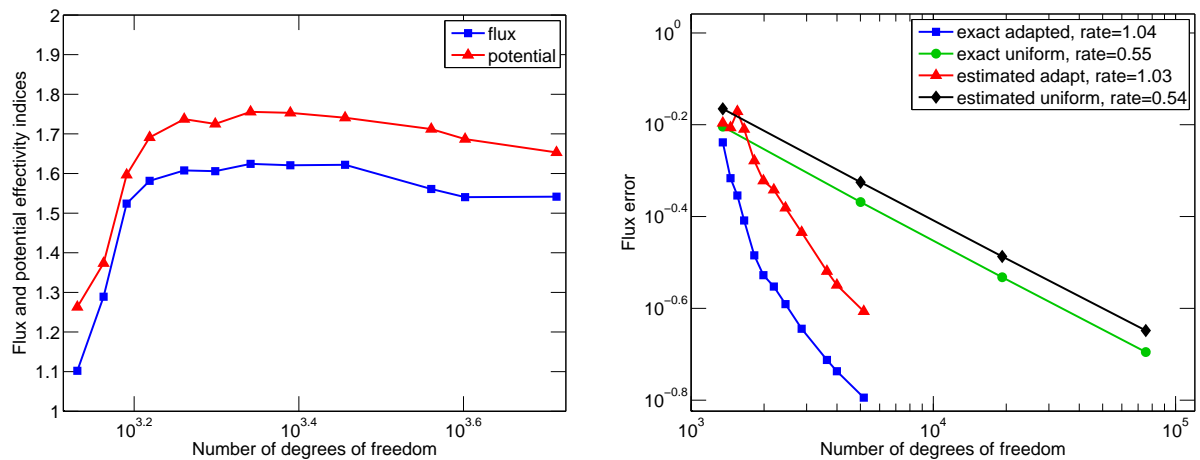

FIG. 7.10. Flux and potential effectivity indices on adaptively refined meshes (left) and estimated and actual flux error (right) using method 1 for Section 7.3

order is approximately $\mathcal{O}\left(h^{0.55}\right)$ for uniform refinement and $\mathcal{O}\left(h^{1.03}\right)$ for the adaptive algorithm which indicates that the refinement resolves the singularity.

8. Concluding remarks. We have introduced in this paper a general framework for guaranteed a posteriori error estimates for multiscale, multinumerics, and mortar coupling. In Section 7, we have classified the various estimators into three methods. Method 1, which uses the $\mathbf{H}(\operatorname{div}, \Omega)$-conforming flux reconstruction $\mathbf{t}_{h}$ following Section 3.3.2, is moderately involved to implement, while requiring the solution of local Neumann problems. It gives the best results for almost all the test cases. Method 2, with $\mathbf{t}_{h}$ constructed following Section 3.3.3, is more expensive but robust in the multiscale setting, under sufficient regularity assumptions. Method 3, where the estimates are given by Corollary 6.1 and where no $\mathbf{H}(\operatorname{div}, \Omega)$-conforming flux reconstruction is used, appears as a cheaper alternative with less sharp results especially in truly multiscale situations.

Appendix A. A technical result for nonmatching meshes. We give here a technical result which was necessary in the proof of Theorem 4.2.

Lemma A.1. Let $\tilde{p}_{h} \in H^{1}\left(\mathcal{T}_{h}\right)$ satisfying Assumptions 3.8 (2)-(3) be given. Then

$$
h_{g}^{-\frac{1}{2}}\left\|\llbracket \tilde{p}_{h} \rrbracket\right\|_{g} \leq C \sum_{T \in \mathcal{T}_{h} ;|g \cap \partial T| \neq 0}\left\|\nabla\left(\tilde{p}_{h}-\psi\right)\right\|_{T}
$$


for all $g \in \mathcal{G}_{h}^{*}$, where $\psi \in H^{1}(\Omega)$ is arbitrary and $C$ depends on $d, \kappa_{\mathcal{T}_{h}}$, and $C_{\mathcal{G}_{h}^{*}}$.

Proof. The proof is a generalization of [2, Theorem 10] to the case where $g \in$ $\mathcal{G}_{h}^{*}$ is a union of sides from $\mathcal{E}_{h}^{\Gamma}$, arising from each side of the interface, such that only $\left\langle\llbracket \tilde{p}_{h} \rrbracket, 1\right\rangle_{g}=0$ holds. If not specified otherwise, $C$ denotes a generic constant depending on $d, \kappa_{\mathcal{T}_{h}}$, and $C_{\mathcal{G}_{h}^{*}}$, not necessarily the same at each occurrence.

Consider $g \in \mathcal{G}_{h}^{*}$ and all the elements $T_{i}^{\prime}$ and $T_{j}^{\prime \prime}$ of $\mathcal{T}_{h}$ from the two subdomains $\Omega_{i}$ and $\Omega_{j}$ such that $g \cap \partial T_{i}^{\prime} \neq \emptyset$ and $g \cap \partial T_{j}^{\prime \prime} \neq \emptyset$, cf. Figure 2.1. Denote $\cup T_{i}^{\prime}$ by $T^{\prime}$ and $\cup T_{j}^{\prime \prime}$ by $T^{\prime \prime}$. Let $T^{*}=T^{\prime}$ or $T^{\prime \prime}$ and consider the following local Neumann problems:

$$
\begin{aligned}
-\Delta \varphi & =0 & & \text { in } T^{*}, \\
\nabla \varphi \cdot \mathbf{n}_{g} & =\llbracket \tilde{p}_{h} \rrbracket & & \text { on } \partial T^{*} \cap g, \\
\nabla \varphi \cdot \mathbf{n}_{T^{*}} & =0 & & \text { on } \partial T^{*} \backslash g, \\
(\varphi, 1)_{T^{*}} & =0 . & &
\end{aligned}
$$

Using Assumption 3.8 (3), it follows that the above problems are well-posed. Let now $\psi \in H^{1}\left(T^{\prime} \cup T^{\prime \prime}\right)$ be arbitrary. Set $\left.\tilde{\psi}\right|_{T^{\prime}}:=\left.\psi\right|_{T^{\prime}}+c_{T^{\prime}}$ and $\left.\tilde{\psi}\right|_{T^{\prime \prime}}:=\left.\psi\right|_{T^{\prime \prime}}+c_{T^{\prime \prime}}$, where $c_{T^{\prime}}:=\left(\tilde{p}_{h}-\psi\right)_{T^{\prime}}$ and $c_{T^{\prime \prime}}:=\left(\tilde{p}_{h}-\psi\right)_{T^{\prime \prime}}$. The function $\psi$ is on $T^{\prime}, T^{\prime \prime}$ shifted by the constants $c_{T^{\prime}}$ and $c_{T^{\prime \prime}}$ so that $\tilde{\psi}$ has the same mean value as $\tilde{p}_{h}$ on both $T^{\prime}, T^{\prime \prime}$, $(\tilde{\psi}, 1)_{T^{\prime}}=\left(\tilde{p}_{h}, 1\right)_{T^{\prime}},(\tilde{\psi}, 1)_{T^{\prime \prime}}=\left(\tilde{p}_{h}, 1\right)_{T^{\prime \prime}}$. Thus, we have

$$
\begin{aligned}
\left(\nabla \varphi, \nabla\left(\tilde{\psi}-\tilde{p}_{h}\right)\right)_{T^{\prime} \cup T^{\prime \prime}}= & \sum_{T \subset T^{\prime} \cup T^{\prime \prime}}\left(\nabla \varphi, \nabla\left(\tilde{\psi}-\tilde{p}_{h}\right)\right)_{T}=\sum_{T \subset T^{\prime} \cup T^{\prime \prime}}\left\langle\nabla \varphi \cdot \mathbf{n}_{T}, \tilde{\psi}-\tilde{p}_{h}\right\rangle_{\partial T} \\
& -\left\|\llbracket \tilde{p}_{h} \rrbracket\right\|_{g}^{2}+\left\langle\llbracket \tilde{p}_{h} \rrbracket, \llbracket \tilde{\psi} \rrbracket\right\rangle_{g}-\sum_{T \subset T^{\prime} \cup T^{\prime \prime}} \mathbf{n}_{T} \cdot \mathbf{n}_{g}\left\langle\llbracket \tilde{p}_{h} \rrbracket, \tilde{\psi}-\tilde{p}_{h}\right\rangle_{\partial T \cap g},
\end{aligned}
$$

using Green's theorem, (A.1a), and noting that $\tilde{p}_{h}$ is by assumption only regular on the elements $T$ of $\mathcal{T}_{h}$. This leads to

$$
\begin{aligned}
\left\|\llbracket \tilde{p}_{h} \rrbracket\right\|_{g}^{2} \leq & \|\nabla \varphi\|_{T^{\prime} \cup T^{\prime \prime}}\left\|\nabla\left(\tilde{\psi}-\tilde{p}_{h}\right)\right\|_{T^{\prime} \cup T^{\prime \prime}}+\sum_{T \subset T^{\prime} \cup T^{\prime \prime}}\left\|\nabla \varphi \cdot \mathbf{n}_{T}\right\|_{-\frac{1}{2}, \partial T}\left\|\tilde{\psi}-\tilde{p}_{h}\right\|_{\frac{1}{2}, \partial T} \\
& +\sum_{T \subset T^{\prime} \cup T^{\prime \prime}}\left\|\llbracket \tilde{p} \tilde{p}_{h} \rrbracket\right\|_{\partial T \cap g}\left\|\tilde{\psi}-\tilde{p}_{h}\right\|_{\partial T \cap g}+\left|\left\langle\llbracket \tilde{p}_{h} \rrbracket, \llbracket \tilde{\psi} \rrbracket\right\rangle_{g}\right|,
\end{aligned}
$$

where $\|\cdot\|_{-\frac{1}{2}, \partial T}$ and $\|\cdot\|_{\frac{1}{2}, \partial T}$ are defined by duality as in $[3,9]$. We now estimate each of the above right-hand-side terms separately.

1) The trace inequality $\|\varphi\|_{g} \leq C h_{g}^{\frac{1}{2}}\|\nabla \varphi\|_{T^{\prime}}$ (recall that (A.1d) holds) gives

$$
\|\nabla \varphi\|_{T^{\prime}}^{2}=\left\langle\nabla \varphi \cdot \mathbf{n}_{T^{\prime}}, \varphi\right\rangle_{\partial T^{\prime}}=\mathbf{n}_{T^{\prime}} \cdot \mathbf{n}_{g}\left\langle\llbracket \tilde{p}_{h} \rrbracket, \varphi\right\rangle_{g} \leq C\left\|\llbracket \tilde{p}_{h} \rrbracket\right\|_{g} h_{g}^{\frac{1}{2}}\|\nabla \varphi\|_{T^{\prime}} .
$$

Proceeding similarly on $T^{\prime \prime}$, it follows that the first term of (A.2) can be bounded by

$$
C\left\|\llbracket \tilde{p}_{h} \rrbracket\right\|_{g} h_{g}^{\frac{1}{2}}\left\|\nabla\left(\tilde{\psi}-\tilde{p}_{h}\right)\right\|_{T^{\prime} \cup T^{\prime \prime}}=C\left\|\llbracket \tilde{p}_{h} \rrbracket\right\|_{g} h_{g}^{\frac{1}{2}}\left\|\nabla\left(\psi-\tilde{p}_{h}\right)\right\|_{T^{\prime} \cup T^{\prime \prime}} .
$$

Here we have also used the fact that $\nabla \tilde{\psi}=\nabla \psi$ (recall $\nabla$ is the piecewise gradient).

2) The second term of (A.2) can be bounded by

$$
\begin{aligned}
& C\|\nabla \varphi\|_{T^{\prime} \cup T^{\prime \prime}}\left(\left\|\nabla\left(\tilde{\psi}-\tilde{p}_{h}\right)\right\|_{T^{\prime} \cup T^{\prime \prime}}^{2}+h_{g}^{-2}\left\|\tilde{\psi}-\tilde{p}_{h}\right\|_{T^{\prime}}^{2}+h_{g}^{-2}\left\|\tilde{\psi}-\tilde{p}_{h}\right\|_{T^{\prime \prime}}^{2}\right)^{\frac{1}{2}} \\
\leq & C\|\nabla \varphi\|_{T^{\prime} \cup T^{\prime \prime}}\left\|\nabla\left(\tilde{\psi}-\tilde{p}_{h}\right)\right\|_{T^{\prime} \cup T^{\prime \prime}},
\end{aligned}
$$


where we have employed the trace theorem and Cauchy-Schwarz's inequality. In addition, we have used the fact that $h_{T}$ and $h_{g}$ are comparable for all $T \subset T^{\prime} \cup T^{\prime \prime}$ and the discrete Poincaré inequality $\left\|\tilde{\psi}-\tilde{p}_{h}\right\|_{T^{*}} \leq C h_{g}\left\|\nabla\left(\tilde{\psi}-\tilde{p}_{h}\right)\right\|_{T^{*}}, T^{*}=T^{\prime}, T^{\prime \prime}$, cf. [30, Theorem 8.1]. The latter result is justified by the Assumption 3.8 (2).

3) Let $T \in \mathcal{T}_{h}$ and $e \in \mathcal{E}_{T}$. Using the trace inequality $\left\|\varphi-\varphi_{T}\right\|_{e} \leq C h_{T}^{\frac{1}{2}}\|\nabla \varphi\|_{T}$,

$$
\left\|\tilde{\psi}-\tilde{p}_{h}\right\|_{\partial T \cap g}^{2} \leq C\left(h_{T}\left\|\nabla\left(\tilde{\psi}-\tilde{p}_{h}\right)\right\|_{T}^{2}+h_{T}^{-1}\left\|\tilde{\psi}-\tilde{p}_{h}\right\|_{T}^{2}\right)
$$

for any $T \subset T^{\prime} \cup T^{\prime \prime}$. Consequently, the third term of (A.2) is bounded by

$$
\begin{aligned}
& C\left\|\llbracket \tilde{p}_{h} \rrbracket\right\|_{g}\left(h_{g}\left\|\nabla\left(\tilde{\psi}-\tilde{p}_{h}\right)\right\|_{T^{\prime} \cup T^{\prime \prime}}^{2}+h_{g}^{-1}\left\|\tilde{\psi}-\tilde{p}_{h}\right\|_{T^{\prime}}^{2}+h_{g}^{-1}\left\|\tilde{\psi}-\tilde{p}_{h}\right\|_{T^{\prime \prime}}^{2}\right)^{\frac{1}{2}} \\
\leq & C\left\|\llbracket \tilde{p}_{h} \rrbracket\right\|_{g} h_{g}^{\frac{1}{2}}\left\|\nabla\left(\tilde{\psi}-\tilde{p}_{h}\right)\right\|_{T^{\prime} \cup T^{\prime \prime}}=C\left\|\llbracket \tilde{p}_{h} \rrbracket\right\|_{g} h_{g}^{\frac{1}{2}}\left\|\nabla\left(\psi-\tilde{p}_{h}\right)\right\|_{T^{\prime} \cup T^{\prime \prime}},
\end{aligned}
$$

using the same argument as in 2).

4) Let us finally turn to the last term of (A.2). We have, using Cauchy-Schwarz's inequality, the fact that $|\llbracket \tilde{\psi} \rrbracket|=\left|c_{T^{\prime}}-c_{T^{\prime \prime}}\right|$, adding and subtracting $\left(\tilde{p}_{h}-\psi\right)_{g}$, and employing the triangle inequality,

$$
\begin{gathered}
\left|\left\langle\llbracket \tilde{p}_{h} \rrbracket, \llbracket \tilde{\psi} \rrbracket\right\rangle_{g}\right| \leq\left\|\llbracket \tilde{p}_{h} \rrbracket\right\|_{g}\left\|c_{T^{\prime}}-c_{T^{\prime \prime}}\right\|_{g} \leq\left\|\llbracket \tilde{p}_{h} \rrbracket\right\|_{g}\left(\left\|\left(\tilde{p}_{h}-\psi\right)_{T^{\prime}}-\left(\tilde{p}_{h}-\psi\right)_{g}\right\|_{g}\right. \\
\left.+\left\|\left(\tilde{p}_{h}-\psi\right)_{T^{\prime \prime}}-\left(\tilde{p}_{h}-\psi\right)_{g}\right\|_{g}\right) .
\end{gathered}
$$

Now, using the same technique as in [32, Lemma 7.2] and employing discrete Friedrichs' inequality [30, Theorem 5.4 and Remark 5.9] $\|\chi\|_{T^{*}} \leq C h_{g}\|\nabla \chi\|_{T^{*}}, T^{*}=T^{\prime}, T^{\prime \prime}$, with $\chi:=\left(\tilde{p}_{h}-\psi\right)-\left(\tilde{p}_{h}-\psi\right)_{g}$, (it is again crucial that Assumption 3.8 (2) holds), we have

$$
\left|\left\langle\llbracket \tilde{p}_{h} \rrbracket, \llbracket \tilde{\psi} \rrbracket\right\rangle_{g}\right| \leq C\left\|\llbracket \tilde{p}_{h} \rrbracket\right\|_{g} h_{g}^{\frac{1}{2}}\left\|\nabla\left(\psi-\tilde{p}_{h}\right)\right\|_{T^{\prime} \cup T^{\prime \prime}} .
$$

Combining the above estimates on the individual terms of (A.2), we come to $\left\|\llbracket \tilde{p}_{h} \rrbracket\right\|_{g} \leq C h_{g}^{\frac{1}{2}}\left\|\nabla\left(\psi-\tilde{p}_{h}\right)\right\|_{T^{\prime} \cup T^{\prime \prime}}$, whence the assertion of the lemma follows.

\section{REFERENCES}

[1] Aarnes, J. E., And Efendiev, Y. An adaptive multiscale method for simulation of fluid flow in heterogeneous porous media. Multiscale Model. Simul. 5, 3 (2006), 918-939.

[2] Achdou, Y., Bernardi, C., And Coquel, F. A priori and a posteriori analysis of finite volume discretizations of Darcy's equations. Numer. Math. 96, 1 (2003), 17-42.

[3] Agmon, S. Lectures on elliptic boundary value problems. Prepared for publication by B. Frank Jones, Jr. with the assistance of George W. Batten, Jr. Van Nostrand Mathematical Studies, No. 2. D. Van Nostrand Co., Inc., Princeton, N.J.-Toronto-London, 1965.

[4] Ainsworth, M. A posteriori error estimation for discontinuous Galerkin finite element approximation. SIAM J. Numer. Anal. 45, 4 (2007), 1777-1798.

[5] Ainsworth, M., And Rankin, R. Fully computable error bounds for discontinuous Galerkin finite element approximations on meshes with an arbitrary number of levels of hanging nodes. SIAM J. Numer. Anal. 47, 6 (2010), 4112-4141.

[6] Arbogast, T., And Chen, Z. On the implementation of mixed methods as nonconforming methods for second-order elliptic problems. Math. Comp. 64, 211 (1995), 943-972.

[7] Arbogast, T., Cowsar, L. C., Wheeler, M. F., and Yotov, I. Mixed finite element methods on nonmatching multiblock grids. SIAM J. Numer. Anal. 37, 4 (2000), 1295-1315.

[8] Arbogast, T., Pencheva, G., Wheeler, M. F., And Yotov, I. A multiscale mortar mixed finite element method. Multiscale Model. Simul. 6, 1 (2007), 319-346.

[9] Bergh, J., And Löfström, J. Interpolation spaces. An introduction. Springer-Verlag, Berlin, 1976. Grundlehren der Mathematischen Wissenschaften, No. 223.

[10] Bernardi, C., And Hecht, F. Error indicators for the mortar finite element discretization of the Laplace equation. Math. Comp. 71, 240 (2002), 1371-1403. 
[11] Bernardi, C., Rebollo, T. C., Hecht, F., and Mghazli, Z. Mortar finite element discretization of a model coupling Darcy and Stokes equations. M2AN Math. Model. Numer. Anal. 42, 3 (2008), 375-410.

[12] Brezzi, F., And Fortin, M. Mixed and hybrid finite element methods, vol. 15 of Springer Series in Computational Mathematics. Springer-Verlag, New York, 1991.

[13] Carstensen, C., And Hu, J. A unifying theory of a posteriori error control for nonconforming finite element methods. Numer. Math. 10\%, 3 (2007), 473-502.

[14] Cochez-Dhondt, S., And Nicaise, S. Equilibrated error estimators for discontinuous Galerkin methods. Numer. Methods Partial Differential Equations 24, 5 (2008), 1236-1252.

[15] Creusé, E., And NicAise, S. A posteriori error estimations of a coupled mixed and standard Galerkin method for second order operators. J. Comput. Appl. Math. 213, 1 (2008), 35-55.

[16] Ern, A., Nicaise, S., And Vohralík, M. An accurate H(div) flux reconstruction for discontinuous Galerkin approximations of elliptic problems. C. R. Math. Acad. Sci. Paris 345, 12 (2007), 709-712.

[17] ERn, A., AND Vohralík, M. Flux reconstruction and a posteriori error estimation for discontinuous Galerkin methods on general nonmatching grids. C. R. Math. Acad. Sci. Paris $347,7-8$ (2009), 441-444.

[18] Ganis, B., And Yotov, I. Implementation of a mortar mixed finite element method using a multiscale flux basis. Comput. Methods Appl. Mech. Engrg. 198 (2009), 3989-3998.

[19] Girault, V., Sun, S., Wheeler, M. F., and Yotov, I. Coupling discontinuous Galerkin and mixed finite element discretizations using mortar finite elements. SIAM J. Numer. Anal. 46, 2 (2008), 949-979.

[20] Glowinski, R., And Wheeler, M. F. Domain decomposition and mixed finite element methods for elliptic problems. In First International Symposium on Domain Decomposition Methods for Partial Differential Equations (Paris, 1987). SIAM, Philadelphia, 1988, pp. 144-172.

[21] Karakashian, O. A., And Pascal, F. A posteriori error estimates for a discontinuous Galerkin approximation of second-order elliptic problems. SIAM J. Numer. Anal. 41, 6 (2003), $2374-2399$.

[22] KIM, K. Y. A posteriori error estimators for locally conservative methods of nonlinear elliptic problems. Appl. Numer. Math. 57, 9 (2007), 1065-1080.

[23] Larson, M. G., And MÅlqvist, A. Adaptive variational multiscale methods based on a posteriori error estimation: energy norm estimates for elliptic problems. Comput. Methods Appl. Mech. Engrg. 196, 21-24 (2007), 2313-2324.

[24] Nicaise, S. A posteriori error estimations of some cell-centered finite volume methods. SIAM J. Numer. Anal. 43, 4 (2005), 1481-1503.

[25] Ohlberger, M. A posteriori error estimates for the heterogeneous multiscale finite element method for elliptic homogenization problems. Multiscale Model. Simul. 4, 1 (2005), 88-114.

[26] Pencheva, G. V., Vohralík, M., Wheeler, M. F., And Wildey, T. Robust a posteriori error control and adaptivity for multiscale, multinumerics, and mortar coupling. Preprint R10015, Laboratoire Jacques-Louis Lions \& ICES Report 10-15, 2010.

[27] Prager, W., And Synge, J. L. Approximations in elasticity based on the concept of function space. Quart. Appl. Math. 5 (1947), 241-269.

[28] Rivière, B., And Wheeler, M. F. A posteriori error estimates for a discontinuous Galerkin method applied to elliptic problems. Comput. Math. Appl. 46, 1 (2003), 141-163.

[29] Roberts, J. E., And Thomas, J.-M. Mixed and hybrid methods. In Handbook of Numerical Analysis, Vol. II. North-Holland, Amsterdam, 1991, pp. 523-639.

[30] Vohralík, M. On the discrete Poincaré-Friedrichs inequalities for nonconforming approximations of the Sobolev space $H^{1}$. Numer. Funct. Anal. Optim. 26, 7-8 (2005), 925-952.

[31] Vohralík, M. A posteriori error estimates for lowest-order mixed finite element discretizations of convection-diffusion-reaction equations. SIAM J. Numer. Anal. 45, 4 (2007), 1570-1599.

[32] Vohralík, M. Residual flux-based a posteriori error estimates for finite volume and related locally conservative methods. Numer. Math. 111, 1 (2008), 121-158.

[33] Vohralík, M. Unified primal formulation-based a priori and a posteriori error analysis of mixed finite element methods. Math. Comp. 79, 272 (2010), 2001-2032.

[34] Wheeler, M., Wildey, T., And Yotov, I. A multiscale preconditioner for stochastic mortar mixed finite elements. Comput. Methods Appl. Mech. Engrg. 200, 9-12 (2011), 1251-1262.

[35] Wheeler, M. F., And Yotov, I. A posteriori error estimates for the mortar mixed finite element method. SIAM J. Numer. Anal. 43, 3 (2005), 1021-1042.

[36] Wohlmuth, B. I. A residual based error estimator for mortar finite element discretizations. Numer. Math. 84, 1 (1999), 143-171. 